\title{
FORMING PLANETESIMALS IN SOLAR AND EXTRASOLAR NEBULAE
}

\author{
E. Chiang ${ }^{1,2}$ And A. Youdin ${ }^{3}$ \\ 1. Department of Astronomy, 601 Campbell Hall, University of California, \\ Berkeley CA 94720, USA; email: echiang@astro.berkeley.edu \\ 2. Department of Earth and Planetary Science, 307 McCone Hall, University of \\ California, Berkeley CA 94720, USA \\ 3. Canadian Institute for Theoretical Astrophysics, University of Toronto, 60 \\ Saint George St., Toronto ON M5S 3H8, Canada; email: youd@cita.utoronto.ca
}

Key Words planet formation, Solar System, accretion, planets, circumstellar disks, fluid mechanics, turbulence

\begin{abstract}
Planets are built from planetesimals: solids larger than a kilometer which grow by colliding pairwise. Planetesimals themselves are unlikely to form by two-body collisions; sub-km objects have gravitational fields individually too weak, and electrostatic attraction is too feeble for growth beyond a few $\mathrm{cm}$. We review the possibility that planetesimals form when self-gravity brings together vast ensembles of small particles. Even when self-gravity is weak, aerodynamic processes can accumulate solids relative to gas, paving the way for gravitational collapse. Particles pile up as they drift radially inward. Gas turbulence stirs particles, but can also seed collapse by clumping them. While the feedback of solids on gas triggers vertical shear instabilities that obstruct self-gravity, this same feedback triggers streaming instabilities that strongly concentrate particles. Numerical simulations find that solids $\sim 10-100 \mathrm{~cm}$ in size gravitationally collapse in turbulent disks. We outline areas for progress, including the possibility that still smaller objects self-gravitate.
\end{abstract}

\section{CONTENTS}

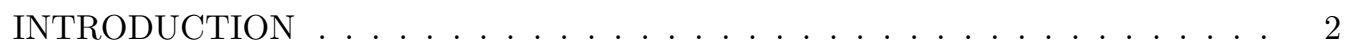

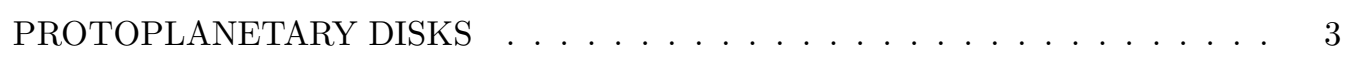

AERODYNAMICS OF INDIVIDUAL PARTICLES . . . . . . . . . . . . . 6

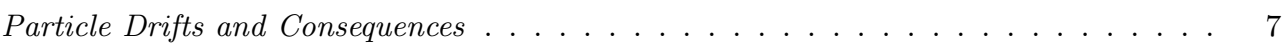

Turbulent Stirring . . . . . . . . . . . . . . . . . . 8

PARTICLE GROWTH BY STICKING . . . . . . . . . . . . . . . . . . . . . 11

CRITERIA FOR GRAVITATIONAL INSTABILITY . . . . . . . . . . . . . . 12

Dynamical Collapse . . . . . . . . . . . . . . . . . . . . . . . . . . . . . . . . . 12

Drag-Assisted Gravitational Instability . . . . . . . . . . . . . . . . . . 14 
THIN DUST LAYERS: VERTICAL SHEARING

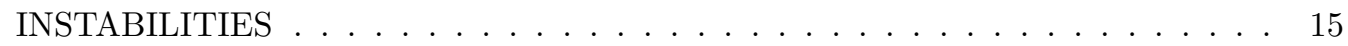

DRAG INSTABILITIES . . . . . . . . . . . . . . . . . . . . 17

Secular Instability of the Dust Layer . . . . . . . . . . . . . . . . . . . . . . . . . . . . 17

Streaming Instability . . . . . . . . . . . . . . . . . . . . . . . . . . 19

GRAVITATIONAL COLLAPSE INTO THE NONLINEAR REGIME . . . . . . . 20

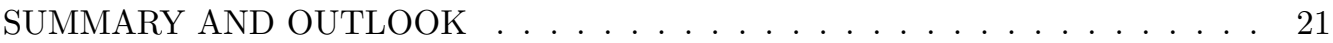

\section{INTRODUCTION}

The nebular hypothesis - that planets coalesce from disks of gas and dust orbiting young stars - is confirmed today in broad outline. Protoplanetary disks of ages 1-10 Myr are now studied routinely (Figure1, Watson et al. 2007). They contain enough mass to spawn planets like those detected around hundreds of Gyr-old stars (Jones et al. 2008). Debris disks, observable via dust generated from collisions between larger parent bodies, bridge our understanding in the 10-100 Myr interval (Wyatt 2008). These parent bodies may represent first-generation planetesimals, the building blocks of planets. Our Solar System may preserve a record of planet formation, in the size distributions of asteroids (Morbidelli et al. 2009) and Kuiper belt objects (Pan \& Sari 2005).

The remarkable journey that disk solids make in growing from microscopic dust to Earth-mass $\left(M_{\oplus}\right)$ planets divides into three legs. At the smallest sizes $\lesssim \mathrm{cm}$, chemical bonds and van der Waals forces enable grains to stick to one another. At the largest sizes $\gg \mathrm{km}$, gravity promotes growth. Pairs of objects coagulate upon colliding because their gravity is strong enough to retain collision fragments.

At intermediate sizes lies the domain of planetesimal formation. It presents the most challenging of terrains; here grain surfaces are insufficiently sticky and the gravity of a single object too feeble. Safronov (1969) kindled the hope that where the individual fails, the collective might succeed: if solid particles have a large enough density en masse, their gravity can draw them together. The many calculations seeking to realize this vision are the subject of our review.

An incompressible fluid body in hydrostatic equilibrium, orbiting a star of mass $M_{*}$ at distance $r$, has sufficient self-gravity to resist disruption by the star's tidal gravitational field if its density exceeds the Roche value $\rho_{\text {Roche }} \approx$ $3.5 M_{*} / r^{3}$ (Chandrasekhar 1987). Though we will see that the effects of selfgravity manifest at arbitrarily low densities (\$5.2), the Roche density is a fine benchmark against which to measure progress towards forming planetesimals, and we will use it as such. It is a formidably large density, 2-3 orders of magnitude greater than typical densities at disk midplanes (\$2). Safronov (1969), and independently Goldreich \& Ward (1973, hereafter GW), proposed that densities approaching Roche (actually a factor of 20 smaller, see \$5.1) could be achieved by having dust settle vertically and accumulate in a thin "sublayer" at the midplane. Weidenschilling (1980) pointed out that large densities were not so easily achieved - that turbulence generated by vertical shear across the sublayer would 
halt settling and prevent gravitational instability (GI). GW appreciated that the sublayer would be turbulent, but overlooked the decisive impact of turbulence on the layer's thickness and density.

This stalemate has been broken - indeed the entire playing field redrawn - in the last decade by abandoning three assumptions. First, the height-integrated surface density ratio of dust to gas need not be solar. Enriching the disk in metals, say by photoevaporation of gas (Throop \& Bally 2005, Gorti \& Hollenbach 2009, Ercolano et al. 2009) or by radial drifts of particles (Youdin \& Shu 2002, hereafter YS), can stop vertical shear turbulence from forestalling GI (Sekiya 1998, YS, Chiang 2008). Second, turbulence, of whatever origin, is not always the enemy of GI. Magneto-rotational tubulence is observed in numerical simulations to generate long-lived structures in gas that can trap particles (Fromang \& Nelson 2005, Johansen et al. 2006b). Third, gas drags particles, but by Newton's Third Law, particles also drag gas. Properly accounting for the backreaction of particles on gas leads to powerful drag instabilities that can concentrate particles and seed GI (Goodman \& Pindor 2000, Youdin \& Goodman 2005, Johansen \& Youdin 2007).

This review is organized as follows. Section 2 establishes order-of-magnitude properties of protoplanetary disks using an up-to-date model for the minimummass solar nebula. Attention is paid to primitive chondritic meteorites. Section 3 gives a primer on the aerodynamics of grains, treating gas drag in the "test particle" limit where backreaction is neglected. We discuss how grain dynamics are affected by gas turbulence. Section 4 describes the extent to which grains can grow by sticking. Section 5 rehearses and comments on Toomre's criterion for GI - and how the criterion is removed when self-gravity is combined with gas drag. Section 6 treats sublayer shearing instabilities. Section 7 introduces secular drag instabilities that concentrate particles without recourse to self-gravity. We offer new insights into Goodman \& Pindor's (2000) toy model for drag instabilities. Section 8 outlines the nonlinear outcome of GI, describing breakthrough numerical simulations. Finally, a summary is supplied in Section 9, where we also list a few forefront problems. Our study is peppered throughout with calculations that readers are encouraged to reproduce - and improve.

Our review complements others in the field of planet formation. Blum \& Wurm (2008) do more justice than we have to the growth of particle aggregates by grain-grain sticking. Goldreich et al. (2004) provide a pedagogical and cutting-edge review of protoplanet accretion by gravitationally focussed, pairwise collisions. For a review of planetesimal formation that overlaps ours and covers some topics in greater detail, see the Les Houches lectures by Youdin (2008).

\section{PROTOPLANETARY DISKS}

A starting point for calculations is the minimum-mass solar nebula (MMSN), derived by adding enough $\mathrm{H}$ and He to solar system planets to restore them to solar composition, and spreading the augmented masses into abutting annuli centered on their orbits (e.g., Weidenschilling 1977b). This exercise is uncertain, since estimates of the total "metal" (non-H and non-He) content of Jupiter range from 10 to $42 M_{\oplus}$; for Saturn the range is 15 to $30 M_{\oplus}$ (Guillot 2005). Even if these uncertainties were reduced, it is unlikely our solar system formed strictly from 
the MMSN. The conversion of disk metals into planets cannot be $100 \%$ efficient, and current planetary orbits may differ significantly from their original ones (e.g., Malhotra 1993). Moreover a major theme of this review is that planetesimal formation may require the metal fraction to evolve to values greater than that of the bulk Sun, at least in certain regions of the disk. Thus the utility of the MMSN lies in providing a baseline for discussing real-world complications, and in establishing orders of magnitude.

For numerical estimates in this review, we adopt a disk surface density:

$$
\begin{gathered}
\Sigma_{\mathrm{g}}=2200 F\left(\frac{r}{\mathrm{AU}}\right)^{-3 / 2} \mathrm{~g} \mathrm{~cm}^{-2} \\
\Sigma_{\mathrm{p}}=33 F Z_{\mathrm{rel}}\left(\frac{r}{\mathrm{AU}}\right)^{-3 / 2} \mathrm{~g} \mathrm{~cm}^{-2}
\end{gathered}
$$

where subscripts $\mathrm{g}$ and $\mathrm{p}$ denote gas and particles (condensed metals), respectively. Our MMSN ( $\left.F=1, Z_{\text {rel }}=1\right)$, uses the updated condensate mass fraction for Solar abundances of 0.015 (Lodders 2003). When methane ice sublimates above temperature $T \approx 41 \mathrm{~K}, Z_{\mathrm{rel}}=0.78$; above $\sim 182 \mathrm{~K}$, water and all other ices are lost so that $Z_{\text {rel }}=0.33$ (Lodders 2003). The coefficient for eq. (2) is chosen to give $1 M_{\oplus}$ of solids in an annulus centered on the Earth's orbit when $F=1$ and $Z_{\text {rel }}=0.33$. We invoke values of $Z_{\text {rel }}>1$ to account for various metal enrichment processes, depicted in Figure 2 and discussed throughout this review (e.g., Sekiya 1998, YS, Throop \& Bally 2005).

Integrated to $r=100 \mathrm{AU}$, eq. (1) yields a mass of $0.03 F M_{\odot}$. Astronomical observations of disks orbiting Myr-old, Sun-like stars suggest they contain 0.001$0.1 M_{\odot}=1-100 M_{\mathrm{J}}$ of gas and dust (Andrews \& Williams 2005). Disk masses are derived from mm-wave radiation from dust, located $\sim 100$ AU from host stars. From the dust emission is calculated a dust mass, and from the dust mass a gas mass is extrapolated assuming a solar dust-to-gas ratio. The gas mass thus imputed is uncertain because the disk metallicity is not known, and because the modeled dust mass depends on an unknown grain size distribution. Disk masses may be systematically underestimated because grains may have grown to sizes $\gg \mathrm{mm}$ and would therefore be practically invisible at sub-cm wavelengths (e.g., Hartmann et al. 2006).

Based on their near-ultraviolet excess emission (Calvet \& Gullbring 1998), young Sun-like stars accrete gas from disks at a typical rate $\dot{M}_{*} \sim 10^{-8} M_{\odot} \mathrm{yr}^{-1}$ (e.g., Hartmann et al. 2006). Accretion implies that disk gas cannot everywhere be static ("passive"). Mass is transported inward by the outward transport of angular momentum, either by turbulence or by ordered flows (Frank et al. 2002). The transport mechanism - or, as is commonly stated, the origin of disk viscosity remains obscure, with proposals ranging from turbulence driven by the magnetorotational instability (MRI; Balbus 2009) to vortices (Lithwick 2009) to gravitational torques (Vorobyov \& Basu 2008). When transport is local, our ignorance is encapsulated in the parameter $\alpha$ (e.g., Frank et al. 2002), the ratio of the local shear stress to the total pressure. Crude, disk-averaged values of $\alpha \sim 10^{-2}$ are inferred from observations of $\dot{M}_{*}$ vs. age (Calvet et al. 2000).

Accretion may be restricted to certain radii and times, as in models that rely upon the MRI - not all of the disk may be sufficiently ionized to couple to magnetic fields (e.g., Bai \& Goodman 2009, see also Chiang \& Murray-Clay 2007). 
Magnetically inert regions are called "dead zones" (Gammie 1996). Whether planetesimals form in active or passive regions of the disk is an outstanding question. This review will explore both cases.

The fraction of stars with near-infrared excess emission attributable to optically thick, gaseous disks decreases from near unity at stellar ages $\lesssim 1 \mathrm{Myr}$, to $\lesssim 5 \%$ at z 10 Myr (Hernández et al. 2008, Hillenbrand 2005). Whatever combination of accretion, photoevaporation, and planet formation is responsible for this observed decline, giant planets - including ice giants, which contain more hydrogen than can be explained by accretion of hydrated solids (Lissauer \& Stevenson 2007) must form within several Myr before the gas dissipates. The same deadline characterizes planetesimal formation, since the ice giants, which by mass are 80-90\% rock and ice, coagulated from planetesimals.

Current models set the formation of solar system giant planets within a disk with more solids, and sometimes more gas, than the MMSN. Goldreich et al. (2004) identify Neptune and Uranus with "isolation-mass" oligarchs at $r \approx 20-30 \mathrm{AU}$, requiring $F Z_{\text {rel }} \approx 61$ Lissauer et al. (2009) prefer $F Z_{\text {rel }} \approx 3.5$ at $r \approx 5 \mathrm{AU}$, so that Jupiter's core can accrete its gaseous envelope within 3 Myr.

At the midplane of a passive disk - one heated solely by stellar radiation - the gas temperature, scale height, and density are approximately

$$
\begin{array}{r}
T=120\left(\frac{r}{\mathrm{AU}}\right)^{-3 / 7} \mathrm{~K} \\
h_{\mathrm{g}}=0.022 r\left(\frac{r}{\mathrm{AU}}\right)^{2 / 7} \\
\rho_{\mathrm{g}}=2.7 \times 10^{-9} F\left(\frac{r}{\mathrm{AU}}\right)^{-39 / 14} \mathrm{~g} \mathrm{~cm}^{-3} .
\end{array}
$$

These are adapted from Chiang \& Goldreich (1997), adjusted for a disk obeying (11) -(2), orbiting a young star of mass $M_{*}=1 M_{\odot}$, radius $R_{*}=1.7 R_{\odot}$, and temperature $T_{*}=4350 \mathrm{~K}$. Thus in our MMSN, water ice starts to condense outside $\sim 0.4$ AU, and methane freezes outside $\sim 12$ AU. More detailed models of passive disks are reviewed by Dullemond et al. (2007). Turbulent accretion can give higher midplane temperatures. The density and thermal structure of active disks depends on the assumed viscosity profile, $\alpha(r)$. One model is that of D'Alessio et al. (2001), which assumes $\alpha(r)=$ constant and is tailored to fit broadband spectra and reflected light images. For simplicity, estimates in this review employ (1) -(5) for both active and passive disks.

What do astronomical observations tell us about grain sizes? In disk surface layers directly illuminated by optical light from host stars, grain sizes are 1-10 $\mu \mathrm{m}$, as deduced from mid-infrared silicate emission bands (Natta et al. 2007), and from scattered light images at similar wavelength (McCabe et al. 2003). Surface grains have settled vertically, residing at heights $z$ above the midplane of $1-3 h_{\mathrm{g}}$ (Chiang et al. 2001); for a disk in which dust and gas are well mixed, $z / h_{\mathrm{g}} \approx 4-5$. At disk midplanes, $\mathrm{mm}$ to $\mathrm{cm}$-sized grains are routinely invoked to match $\mathrm{mm}$ to

\footnotetext{
${ }^{1}$ A protoplanet of mass $M_{p}$ becomes "isolated" when it has accreted an annulus of disk material having a width about $5 \times$ the radius of its Hill sphere, $R_{\mathrm{H}} \approx\left[M_{p} /\left(3 M_{*}\right)\right]^{1 / 3} r$ (e.g., Greenberg et al. 1991). The average density of the planet spread through its Hill sphere is of order $\rho_{\text {Roche }} \approx 3.5 M_{*} / r^{3}$.
} 
cm-wave spectra and images (e.g., D'Alessio et al. 2001, Testi et al. 2003).

The most primitive meteorites, of nearly solar photospheric composition except in volatile elements, also offer data on particle sizes. Confounding meteoriticists and astronomers alike is why up to $\sim 90 \%$ of their volume is filled with chondrules: once molten, 0.1-mm to $\mathrm{cm}$-sized spheres that solidified $4.57 \mathrm{Gyr}$ ago (e.g., Hewins 1996). Chondrule petrology is consistent with their having been heated just above liquidus for less than minutes, and having cooled for hours to days. The heating mechanism is not known; nebular shocks are suspected, but the origin of such shocks is debated (Desch et al. 2005). Chondrules might well have been the building blocks of the first-generation planetesimals, brought together by self-gravity. That $\sim 10 \%$ of chondrules are binaries which collided and fused while still partially molten implies that when chondrules were suspended in space, they had large collective densities (Gooding \& Keil 1981), possibly exceeding the Roche value. Extremely dusty environments are also indicated by the retention of volatiles in chondrules (Alexander et al. 2008).

\section{AERODYNAMICS OF INDIVIDUAL PARTICLES}

Gas drags particles. The degree of coupling is measured by the dimensionless stopping time $\tau_{\mathrm{s}} \equiv \Omega_{\mathrm{K}} t_{\mathrm{s}}$, where $\Omega_{\mathrm{K}}$ is the Keplerian angular velocity and

$$
t_{\mathrm{s}} \equiv m v_{\text {rel }} / F_{\mathrm{D}} \approx \begin{cases}\rho_{\mathrm{s}} s /\left(\rho_{\mathrm{g}} c_{\mathrm{g}}\right) & \text { if } s \lesssim 9 \lambda / 4 \text { (Epstein) } \\ 4 \rho_{\mathrm{s}} s^{2} /\left(9 \rho_{\mathrm{g}} c_{\mathrm{g}} \lambda\right) & \text { if } s \gtrsim 9 \lambda / 4, \text { Re } \lesssim 1 \text { (Stokes) }\end{cases}
$$

measures how long it takes a particle of mass $m$, radius $s$, and internal density $\rho_{\mathrm{s}}$ to have its speed $v_{\text {rel }}$ relative to gas be reduced by order unity. The gas sound speed is $c_{\mathrm{g}}$. Particles are well entrained in gas when $\tau_{\mathrm{s}} \ll 1$. We have given the two cases for the drag force $F_{\mathrm{D}}$ most relevant for planetesimal formation: Epstein's (1924) law of free molecular drag, arising from the difference $\sim \rho_{\mathrm{g}}\left[\left(c_{\mathrm{g}}+v_{\text {rel }}\right)^{2}-\left(c_{\mathrm{g}}-v_{\text {rel }}\right)^{2}\right]$ in momentum fluxes received by the windward and leeward faces of the particle, and Stokes drag for low Reynolds number Re $\equiv s v_{\text {rel }} /\left(\lambda c_{\mathrm{g}}\right)$. Given (5), the mean free path for collisions between gas molecules is $\lambda \approx 0.5 F^{-1}(r / \mathrm{AU})^{39 / 14} \mathrm{~cm} 2$ Other cases for $F_{\mathrm{D}}$ are given by Adachi et al. (1976) and Weidenschilling (1977a). For $F=2$ and $\rho_{\mathrm{s}}=1 \mathrm{~g} / \mathrm{cm}^{3}$, marginally coupled bodies $\left(\tau_{\mathrm{s}}=1\right)$ have $s=s_{1}$ increasing from 35 to $120 \mathrm{~cm}$ in the Stokes regime as $r$ runs from 1 to $7 \mathrm{AU}$; thereafter $s_{1}$ declines with $r$ in the Epstein regime, with $s_{1} \approx 13 \mathrm{~cm}$ at $30 \mathrm{AU}$.

Gas and dust move at different velocities because pressure gradients barely accelerate particles with $\rho_{\mathrm{s}} \gg \rho_{\mathrm{g}}$. For the moment, let us neglect turbulence, and assume the collective particle density $\rho_{\mathrm{p}} \ll \rho_{\mathrm{g}}$ so we can ignore the backreaction of dust on gas. Then the azimuthal gas velocity $v_{\mathrm{g} \phi}$ obeys

$$
\frac{v_{\mathrm{g} \phi}^{2}}{r}=\frac{G M_{*}}{r^{2}}-\frac{1}{\rho_{\mathrm{g}}} \frac{\partial P}{\partial r} .
$$

\footnotetext{
${ }^{2}$ We use a constant molecular cross section, $\sigma\left(\mathrm{H}_{2}\right) \approx 2 \times 10^{-15} \mathrm{~cm}^{2}$, that reproduces the dynamical viscosity $\mu$ at $200 \mathrm{~K}$. But constant $\sigma\left(\mathrm{H}_{2}\right)$ assumes $\mu \propto \sqrt{T}$, which fails for $T \lesssim 70$ $\mathrm{K}$, the Sutherland constant for $\mathrm{H}_{2}$. At colder temperatures $\mu \propto T^{3 / 2}$ so that $\sigma\left(\mathrm{H}_{2}\right) \propto \mathrm{T}^{-1}$ (Chapman \& Cowling 1970). For simplicity we neglect the lower values of viscosity and $\lambda$ that this effect would produce in cold regions.
} 
Pressure $P \approx \rho_{\mathrm{g}} c_{\mathrm{g}}^{2}$ makes the gas rotate more slowly than the local Keplerian velocity $v_{\mathrm{K}} \equiv \Omega_{\mathrm{K}} r=\sqrt{G M_{*} / r}$ by

$$
\eta v_{\mathrm{K}} \equiv v_{\mathrm{K}}-v_{\mathrm{g} \phi} \approx-\frac{\partial P / \partial \ln r}{2 \rho_{\mathrm{g}} v_{\mathrm{K}}} \approx 25\left(\frac{r}{\mathrm{AU}}\right)^{1 / 14} \mathrm{~m} \mathrm{~s}^{-1}
$$

using the thin disk approximation $c_{\mathrm{g}} / v_{\mathrm{K}}=h_{\mathrm{g}} / r \ll 1$. While $\eta v_{\mathrm{K}} \sim c_{\mathrm{g}}^{2} / v_{\mathrm{K}}$ is independent of total disk mass and is nearly independent of $r$, it increases in hotter disks, perhaps to $\gtrsim 50 \mathrm{~m} \mathrm{~s}^{-1}$. An individual particle seeks to orbit at the full $v_{\mathrm{K}}$. Thus it normally experiences a headwind, except possibly where there are variations to the power-law descent of $P(r)$, as considered at the end of 33.1 .2 ,

\subsection{Particle Drifts and Consequences}

3.1.1 LAminar Drift SpeEds In a passive disk, a particle settles vertically to the midplane and, on longer timescales, drifts radially inward as the headwind saps its angular momentum. The following is drawn from Youdin (2008, see also Nakagawa et al. 1986). If we neglect backreaction, the cylindrical components of the gas velocity are $v_{\mathrm{g} r}=0, v_{\mathrm{g} z}=0$, and $v_{\mathrm{g} \phi} \equiv(1-\eta) \Omega_{\mathrm{K}} r$. In $z$, the particle's equation of motion reads

$$
\ddot{z}=-\dot{z} / t_{\mathrm{s}}-\Omega_{\mathrm{K}}^{2} z
$$

where the last term accounts for stellar gravity, for $z \ll r$. For $\tau_{\mathrm{s}} \ll 1$, the particle settles at terminal velocity $-\Omega_{\mathrm{K}}^{2} z t_{\mathrm{s}}$. For $\tau_{\mathrm{s}} \gg 1$, the particle behaves as a lightly damped harmonic oscillator whose amplitude decays as $e^{-t /\left(2 t_{\mathrm{s}}\right)}$. A characteristic time for settling, valid for all $\tau_{\mathrm{s}}$, is

$$
t_{z} \sim \frac{1}{\Omega_{\mathrm{K}}}\left(\frac{2 \tau_{\mathrm{s}}^{2}+1}{\tau_{\mathrm{s}}}\right) .
$$

In $r$ and $\phi$,

$$
\begin{array}{r}
\ddot{r}-r \dot{\phi}^{2}=-v_{\mathrm{K}}^{2} / r-\dot{r} / t_{\mathrm{s}} \\
r \ddot{\phi}+2 \dot{r} \dot{\phi}=-\left(r \dot{\phi}-v_{\mathrm{g} \phi}\right) / t_{\mathrm{s}} .
\end{array}
$$

Writing $\dot{\phi}=\Omega_{\mathrm{K}}+\delta v_{\phi} / r$ where $\left|\delta v_{\phi}\right| \ll \Omega_{\mathrm{K}} r$, we approximate $\ddot{\phi} \approx \dot{\Omega}_{\mathrm{K}} \approx$ $-3 \Omega_{\mathrm{K}} \dot{r} /(2 r)$ and drop $|\ddot{r}| \ll\left|\dot{r} / t_{\mathrm{s}}\right|$. Both approximations can be checked a posteriori. To first order in $\delta v_{\phi}$,

$$
\begin{array}{r}
\dot{r} \approx-2 \eta \Omega_{\mathrm{K}} r\left(\frac{\tau_{\mathrm{s}}}{1+\tau_{\mathrm{s}}^{2}}\right) \\
\delta v_{\phi}=\left(\dot{\phi}-\Omega_{\mathrm{K}}\right) r \approx \frac{-\eta \Omega_{\mathrm{K}} r}{1+\tau_{\mathrm{s}}^{2}} .
\end{array}
$$

The time for radial drift, $t_{r} \equiv|r / \dot{r}|$, is $\sim \eta^{-1}$ longer than $t_{z}$. Both times are minimized, and velocities relative to gas are maximized at $\sim \eta v_{\mathrm{K}}$, for marginally coupled bodies. This is the well-known problem that boulders having sizes $\mathrm{s}_{1} \approx m$ drift towards the star in $\min \left(t_{r}\right) \sim\left(\eta \Omega_{\mathrm{K}}\right)^{-1} \sim 200(r / \mathrm{AU})^{13 / 14} \mathrm{yr}$, too quickly to form planets. 
3.1.2 Pileups And Pressure Traps As particles of a given size drift inward, they tend to "pile up," increasing $\Sigma_{\mathrm{p}} / \Sigma_{\mathrm{g}}$ (YS, Youdin \& Chiang 2004); see Figure 2. Their inward mass flux decreases with decreasing $r$ if Epstein drag applies and the outer regions are not already depleted in solids. Idealized pile-ups march inward on the drift timescale with increasing amplitude. The actual ability of particles to pile up coherently will be affected by ongoing evolution of the particle size distribution and by any disk turbulence. Youdin \& Chiang (2004) explore one prescription for turbulent viscosity predicated on the KHI that amplifies pile-ups, but its basis is unproven; see §5.2.3 of Garaud \& Lin (2004). Stepinski \& Valageas (1996) show, by combining the drift and turbulent diffusion of particles (see \$3.2) with the viscous evolution of $\Sigma_{g}$, that the solids-to-gas ratio in a disk evolves, and often increases, as it accretes onto the star.

A special pile-up of dust could occur just inside an ice-line (e.g. Ida \& Lin 2008). When "dirty snowballs" drift towards and evaporate inside the ice-line, they unleash small dust grains that may accumulate there (see Ciesla \& Cuzzi 2006, who included this effect in a coagulation model). Furthermore, the ice-line may act as a "cold trap": vapor that diffuses radially outward and crosses the line will condense and accumulate (Stevenson \& Lunine 1988). Though water is the dominant volatile, the methane condensation front could also be significant (\$2).

We have shown that inward particle drift is a direct consequence of gas pressure decreasing radially outward. By the same physics, solids of all sizes drift into and collect within local pressure maxima (Whipple 1972), with $\tau_{\mathrm{s}} \sim 1$ particles accumulating fastest. If a pressure bump has a narrow radial width $\ell<r$, even small amplitudes $\delta P \sim(\ell / r) P$ will produce local maxima. Proposed sources of pressure bumps include gaseous spiral arms (Rice et al. 2004), anticyclonic vortices (Chavanis 2000), the aforementioned ice-lines, and fluctuations in magnetorotational turbulence (Johansen et al. 2009a, see 93.2.3), including spatial variations in the background flux (Kato et al. 2009) or magnetic resistivity, as at the edges of dead zones (Gammie 1996). Youdin (2008) discusses pressure trapping in more detail.

\subsection{Turbulent Stirring}

The response of solids to turbulent gas is multi-faceted (see Toschi \& Bodenschatz 2009, especially their section 4.1). Over many eddy times, turbulence diffuses solids, vertically and radially. On shorter timescales, transient flow structures can have just the opposite effect: they can collect particles.

Many studies assume that turbulence in disks is Kolmogorov in character, and in our example calculations below we also adopt this view. We associate $\delta v_{\mathrm{o}}$, $t_{\mathrm{o}}$, and $\ell_{\mathrm{o}} \sim \delta v_{\mathrm{o}} t_{\mathrm{o}}$ with the speed, turnover time, and length of the largest and fastest "outer scale" eddies. Smaller eddies of length scale $\ell$ have slower speeds $\delta v_{\ell} \sim \delta v_{\mathrm{o}}\left(\ell / \ell_{\mathrm{o}}\right)^{1 / 3}$ and shorter turnover times $t_{\ell}=\ell / \delta v_{\ell} \propto \ell^{2 / 3}$. The cascade of energy from larger to smaller scales terminates at the "inner scale," characterized by $\ell_{\mathrm{i}} \sim \nu^{3 / 4} t_{\mathrm{o}}^{1 / 4} / \delta v_{\mathrm{o}}^{1 / 2}, t_{\mathrm{i}} \sim \sqrt{\nu t_{\mathrm{o}}} / \delta v_{\mathrm{o}}$, and $\delta v_{\mathrm{i}} \sim \ell_{\mathrm{i}} / t_{\mathrm{i}}$ set by the molecular viscosity $\nu$. For many estimates we assume that outer scale eddies have lifetimes limited by orbital shear, so that $t_{\mathrm{O}} \sim \Omega_{\mathrm{K}}^{-1}$. By ignoring the difference between the turbulent diffusivity for mass, $D_{\mathrm{g}} \sim \delta v_{\mathrm{o}} \ell_{\mathrm{o}} \sim \delta v_{\mathrm{o}}^{2} t_{\mathrm{o}}$, with the turbulent diffusivity for angular momentum, $\alpha c_{\mathrm{g}} h_{\mathrm{g}} \sim \alpha c_{\mathrm{g}}^{2} \Omega_{\mathrm{K}}^{-1}$, we extract the convenient prescriptions 
$\delta v_{\mathrm{o}} \sim \sqrt{\alpha} c_{\mathrm{g}}$ and $\ell_{\mathrm{o}} \sim \sqrt{\alpha} h_{\mathrm{g}}$ (Cuzzi et al. 2001, see also Youdin \& Chiang 2004).

However the assumption of homogeneous, isotropic turbulence characterized by cascades of energy down to smaller scales may be incorrect. Simulations of MRI turbulence, both global (Fromang \& Nelson 2005) and in large shearing boxes (Johansen et al. 2009a), show that inverse cascades produce pressure perturbations that survive many tens of orbits and have in-plane sizes larger than $h_{\mathrm{g}}$. These coherent structures may accumulate particles by pressure trapping (Figure 3, \$3.1.2).

3.2.1 Particle-Particle Velocities In active regions of the disk, relative gas-particle and particle-particle velocities may be dominated by turbulence (Kusaka et al. 1970, Cameron 1973), rather than the systematic drifts considered in 33.1.1. Voelk et al. (1980) derive integral expressions for these relative velocites, evaluating them numerically for Kolmogorov turbulence. Consider identical grains with $t_{\mathrm{i}}<t_{\mathrm{s}}<t_{\mathrm{o}}$, i.e. stopping times between the inner and outer scales. Collision speeds are acquired from eddies with a turnover time $t_{\ell} \sim t_{\mathrm{s}}$. If $t_{\ell} \ll t_{\mathrm{s}}$, coupling to the eddy is too weak to excite particle motion. If $t_{\ell} \gg t_{\mathrm{s}}$, grains are so tightly coupled to the incompressible gas flow that they do not collide. Thus colliding grains have relative velocity $\delta v_{\mathrm{p}, \mathrm{p}} \sim \delta v_{\ell} \sim \delta v_{\mathrm{o}}\left(t_{\mathrm{s}} / t_{\mathrm{o}}\right)^{1 / 2}$ which is not to be confused with the random speed of an individual particle, $\delta v_{\mathrm{p}} \sim \delta v_{\mathrm{o}}$.

For $t_{\mathrm{s}}>t_{\mathrm{o}}$, particles are loosely coupled to all eddies. During $t_{\mathrm{s}}$ there are $t_{\mathrm{S}} / t_{\mathrm{o}}$ kicks from the strongest, outer scale eddies, so the random walk in particle velocity saturates at $\delta v_{\mathrm{p}} \sim \delta v_{\mathrm{o}} / \sqrt{t_{\mathrm{s}} / t_{\mathrm{o}}}$ (Youdin \& Lithwick 2007). Since the random walks of different particles are uncorrelated, the random velocity is now also the typical relative speed.

Combining our expressions for collision speeds gives

$$
\delta v_{\mathrm{p}, \mathrm{p}} \sim \delta v_{\mathrm{o}} \sqrt{\mathrm{St} /\left(1+\mathrm{St}^{2}\right)}
$$

where the Stokes number St $\equiv t_{s} / t_{o}$. This agrees roughly with Voelk et al. (1980). Weidenschilling (1984) provides more precise fitting formulae - further refined by Ormel \& Cuzzi (2007) - that include important corrections for $t_{\mathrm{s}}<t_{\mathrm{i}}$ and unequal particle sizes. Youdin \& Lithwick (2007) confirm that orbital dynamics introduces only modest corrections when $t_{\mathrm{o}} \lesssim \Omega_{\mathrm{K}}^{-1}$.

Thus collision speeds in disks peak at $\delta v_{\mathrm{o}}$ for $\tau_{\mathrm{s}} \sim 1$ particles, since St $\sim \tau_{s}$ when $t_{\mathrm{o}} \sim \Omega_{\mathrm{K}}^{-1}$. Magneto-rotational turbulence with $\delta v_{\mathrm{o}} \sim \sqrt{\alpha} c_{\mathrm{g}} \sim 70\left(\alpha / 10^{-2}\right)^{1 / 2}$

$(r / \mathrm{AU})^{-3 / 14} \mathrm{~m} \mathrm{~s}^{-1}$ can induce collision speeds faster than drift speeds (eqs. 13 and (14).

3.2.2 Diffusion and Particle Scale Height On timescales $>t_{\mathrm{o}}$, turbulence diffuses particles spatially. Youdin \& Lithwick (2007) compute, formally and by order-of-magnitude methods, the particle diffusivity $D_{\mathrm{p}}$ as a function of the gas mass diffusivity $D_{\mathrm{g}}$. When $\tau_{\mathrm{s}}$ is small, orbital dynamics are negligible. If further the Stokes number St $\equiv t_{\mathrm{S}} / t_{\mathrm{o}}<1$, then particles are well coupled to gas and $D_{\mathrm{p}} \sim D_{\mathrm{g}}$. If $\mathrm{St}>1$, it takes $t_{\mathrm{s}}$ for the loosely coupled particle to have its random velocity $\delta v_{\mathrm{p}}$ (derived in the paragraph just above eq. 15) changed by order unity. Then $D_{\mathrm{p}} \sim \delta v_{\mathrm{p}}^{2} t_{\mathrm{s}} \sim \delta v_{\mathrm{o}}^{2} t_{\mathrm{o}} \sim D_{\mathrm{g}}$. Thus when $\tau_{\mathrm{s}} \lesssim 1, D_{\mathrm{p}} \sim D_{\mathrm{g}}$ regardless of St. In general, $D_{\mathrm{p}} \sim D_{\mathrm{g}} /\left(1+\tau_{\mathrm{s}}^{2} / 4\right)$ because orbital epicycles limit 
diffusion (Youdin \& Lithwick 2007).

The time for particles to diffuse radially $r^{2} / D_{\mathrm{p}}$ is shorter than the drift time $t_{r}$ when $\alpha>\tau_{\mathrm{s}}$. Thus for $\alpha \sim 10^{-2}$, sub-cm-sized particles are well coupled to the disk accretion flow; but see Takeuchi \& Lin (2002) for possible height-dependent complications.

By equating the vertical diffusion time $h_{\mathrm{p}}^{2} / D_{\mathrm{p}}$ with the gravitational settling time $t_{z}$, we find a turbulent dust scale height

$$
h_{\mathrm{p}} \sim \sqrt{\frac{D_{\mathrm{g}}}{\Omega_{\mathrm{K}} \tau_{\mathrm{s}}}} \sim \sqrt{\frac{\alpha}{\tau_{\mathrm{s}}}} h_{\mathrm{g}}
$$

for all $\tau_{\mathrm{s}}$. Carballido et al. (2006) derive and numerically confirm (16) for $\tau_{\mathrm{s}} \gg 1$. The $\tau_{\mathrm{s}} \ll 1$ limit is well-known (Cuzzi et al. 1993, Dubrulle et al. 1995), but see the end of 3.2 .3 for a possible correction.

3.2.3 Turbulent Concentration Between Eddies On timescales < $t_{\mathrm{o}}$, particles tend to be centrifugally flung out of high vorticity eddies, and funneled into their interstices of lower vorticity (Maxey 1987, Eaton \& Fessler 1994, Toschi \& Bodenschatz 2009). Since centrifugal support in eddy vortices makes them low pressure centers, turbulent concentration is consistent with the tendency of particles to seek high pressure (\$3.1.2, Youdin 2008). Particles of given $t_{\mathrm{s}}$ are concentrated preferentially by eddies that turn over on the same timescale; in other words, particles are concentrated most strongly by eddies to which they are marginally coupled. In a Kolmogorov cascade, smaller eddies are more effective than larger eddies at concentrating their respective, marginally coupled particles. This is because vorticity $\sim \delta v_{\ell} / \ell \propto \ell^{-2 / 3}$, down to the dissipation scale where $\ell_{\mathrm{i}} \sim 5(r / \mathrm{AU})^{135 / 56} \mathrm{~m}$ and $t_{\mathrm{i}} \sim 30(r / \mathrm{AU})^{9 / 4} \mathrm{~s}$ for $F=2, \alpha \sim 0.01$, and $t_{\mathrm{o}} \sim \Omega_{\mathrm{K}}^{-1}$. Turbulent concentration is special since it can collect $\tau_{\mathrm{s}} \ll 1$ particles. Those particles that are optimally concentrated are marginally coupled to the smallest eddies: they have $t_{\mathrm{s}}=t_{\mathrm{i}}$, which corresponds to $s=s_{\mathrm{i}} \sim 0.1 \mathrm{~mm}$ at $1 \mathrm{AU}$ and $s_{\mathrm{i}} \sim 10 \mu \mathrm{m}$ at $30 \mathrm{AU}$, with some uncertainty in the viscosity (see footnote 2).

Cuzzi et al. (2001) apply turbulent concentration to chondrules: objects having sizes $0.1-1 \mathrm{~mm}$ are optimally concentrated at $r=2.5 \mathrm{AU}$ in their model disk, consistent with our estimate of $s_{\mathrm{i}}$ above. The shape of the chondrule size distribution is well reproduced by turbulent concentration. This raises two interesting possibilities: (1) the chondrule size distribution was initially much wider, and has since been narrowed to the one observed today by turbulent concentration; or less likely (2) chondrules happen to form with sizes that closely match optimal concentration scales at the location of the asteroid belt.

The upper limit on concentration is set by mass loading, the backreaction of particles on the turbulence. In direct numerical simulations of $s_{\mathrm{i}}$-sized particles in turbulent gas, Hogan \& Cuzzi (2007) find a maximum concentration factor $\Phi \equiv$ $\rho_{\mathrm{p}} / \rho_{\mathrm{g}} \approx 100$ at the grid, i.e. dissipation scale. This maximum value obtains for moderate Reynolds numbers $\operatorname{Re}_{\mathrm{o}}=\ell_{\mathrm{o}} \delta v_{\mathrm{o}} / \nu$ and a box-averaged $\langle\Phi\rangle=1$. Extrapolating these results using "cascade multipliers" (Sreenivasan \& Stolovitzky 1995), they show that $\max \Phi \approx 100$ still applies at higher $\mathrm{Re}_{\mathrm{o}}$. Maximal clumping at the dissipation scale yields a mass $\Phi \rho_{\mathrm{g}} \ell_{\mathrm{i}}^{3}$ equal to that contained in a compact $10-\mathrm{cm}$ solid at $r=2.5 \mathrm{AU}$. However these clumps are unlikely to compactify because of their extremely short characteristic lifetimes, on the order of $t_{\mathrm{i}}$ 
(Eaton \& Fessler 1994). For our disk parameters, $t_{\mathrm{i}} \sim 4$ minutes.

Cuzzi et al. (2008, hereafter CHS08) introduce the possibility that $s_{\mathrm{i}}$-sized solids concentrate on much larger length scales $\ell_{*} \sim 10^{4} \mathrm{~km}$, or $10^{5} \ell_{\mathrm{i}}$ at $r=2.5 \mathrm{AU}$. The clump mass $\sim \Phi \rho_{\mathrm{g}} \ell_{*}^{3}$ would correspond to a compact $\sim 20-\mathrm{km}$ planetesimal, for $\Phi \sim 100$ concentration. Large scale fluctuations might arise as an "intermittent" phenomenon: deviations from self-similarity that show up in high order structure functions (Frisch 1996). Evidence exists for particle clumping at larger scales, i.e. in the inertial range of isotropic turbulence, but preferentially for particles larger than $s_{\mathrm{i}}$, with $t_{\mathrm{s}}>t_{\mathrm{i}}$ (Bec et al. 2007). This is to be expected since eddies in the inertial range have turnover times longer than $t_{\mathrm{i}}$. Whether clumping of $s_{i}$-sized or larger particles happens at astrophysically interesting amplitudes and rates is an active area of research.

CHS08 find that an $\ell_{*}$-scale clump is massive enough to survive ram pressure stripping as it plows through gas. They propose that self-gravity draws solids to the clump center at the terminal velocity, a process that lasts $\sim 100$ orbits. The turbulence that is invoked to create the clump could still destroy it, since the lifetime of an $\ell_{*}$-eddy at $2.5 \mathrm{AU}$ is $t_{*} \sim t_{\mathrm{o}}\left(\ell_{*} / \ell_{\mathrm{o}}\right)^{2 / 3} \sim 0.007$ orbit. Streaming instabilities (\$7.2) may aid in clump survival, as they show that mass loading can promote particle clumping in Keplerian disks. The enticing possibility that chondrules comprise first-generation planetesimals warrants further investigation in this area.

Eq. (16) for the turbulent dust scale height might need revision because turbulent concentration accelerates vertical settling (Maxey 1987). The correction has not been investigated, but would apply for St $\ll 1$. Turbulent concentration is also neglected in studies of grain growth by sticking, a subject to which we now turn.

\section{PARTICLE GROWTH BY STICKING}

Particles stick upon colliding if they move slowly enough, dissipate enough energy during impact, and are small enough - since surface area increases relative to mass for smaller bodies. We can estimate the relevant orders of magnitude by comparing the initial kinetic energy of two identical elastic spheres colliding at speed $v_{\text {col }}$, with the surface binding energy at the moment of maximum deformation. From Hertz's law of contact, the radius of the compressed cap is $b \sim s\left(\rho_{\mathrm{s}} v_{\mathrm{col}}^{2} / E\right)^{1 / 5}$, where $E$ is Young's modulus. The binding energy is $\sim \gamma b^{2}$, where $\gamma$ is the surface tension from unsaturated bonds, and exceeds the collisional energy for

$$
\begin{array}{r}
v_{\text {col }}<v_{\text {stick }} \sim 2.8\left(\frac{\gamma}{370 \mathrm{erg} \mathrm{cm}^{-2}}\right)^{5 / 6}\left(\frac{7 \times 10^{10} \mathrm{erg} \mathrm{cm}^{-3}}{E}\right)^{1 / 3} \\
\times\left(\frac{1 \mathrm{~g} \mathrm{~cm}^{-3}}{\rho_{\mathrm{s}}}\right)^{1 / 2}\left(\frac{\mu \mathrm{m}}{s}\right)^{5 / 6} \mathrm{~m} \mathrm{~s}^{-1}
\end{array}
$$

for ice (Chokshi et al. 1993, Youdin 2004). For $\mu \mathrm{m}$-sized spheres made of silicate, (17) yields $v_{\text {stick }} \sim 7 \mathrm{~cm} \mathrm{~s}^{-1}$, about an order of magnitude smaller than sticking velocities measured experimentally (Blum \& Wurm 2008). 
In laminar disks, growth by sticking is expected to stall at $\sim \mathrm{cm}$ sizes. From equation (13), radial drift velocities exceed $\sim 1 \mathrm{~m} \mathrm{~s}^{-1}$ - the sticking velocity for $\mu \mathrm{m}$-sized monomers - once $\tau_{\mathrm{s}} \sim 0.02$, corresponding to particle sizes $s \sim 5 \mathrm{~cm}$, $4 \mathrm{~cm}$, and $2 \mathrm{~mm}$ at 1,5 , and $30 \mathrm{AU}$, respectively, for $F=2$. Thus super$\mathrm{cm}$ particles would fail to accrete smaller grains that move with the gas. As judged by equation (17), comparably-sized, super-cm bodies would stick only at unrealistically low relative speeds, with extremely low surface binding energies per unit mass. Indeed bodies $z \mathrm{~mm}$ in size have not been observed to stick experimentally (Blum \& Wurm 2008).

Particle porosity abets sticking by allowing for greater dissipation of kinetic energy (Dominik \& Tielens 1997). Moreover porosity lowers $\rho_{\mathrm{s}}$, which permits larger masses to coagulate when growth is limited to a fixed $\tau_{\mathrm{s}}$, as above. The degree of enhanced mass growth depends on the drag regime; fixing the stopping time gives a particle mass $m \propto \rho_{\mathrm{S}}^{-2}$ for Epstein drag, and $m \propto 1 / \sqrt{\rho_{\mathrm{S}}}$ for Stokes drag. These advantages are limited, however, since collisions at speeds of $0.1-$ $1 \mathrm{~m} \mathrm{~s}^{-1}$ restructure and compactify aggregates composed of $\mu \mathrm{m}$-sized monomers (Dominik \& Tielens 1997).

Simulations of grain growth by Dullemond \& Dominik (2005) and Ormel et al. (2007) include vertical settling and neglect radial drifts. Dullemond \& Dominik (2005) find that in laminar disks, porous aggregates achieve equivalent compact sizes of a few $\mathrm{cm}$ before settling to the midplane, while particles assumed to be always compact grow up to $1 \mathrm{~cm}$ (see their Figure 3, models S2, S5, and S6). Ormel et al. (2007) calculate that in turbulent disks, porous bodies attain equivalent compact sizes of several $\mathrm{cm}$, for $\alpha=10^{-2}$ at $r=1$ and $5 \mathrm{AU}$.

In both these studies, $\mu$ m-sized grains deplete, and the disk becomes optically thin, within $\sim 10^{3} \mathrm{yr}$. This timescale is too short to be reconciled with astronomical observations of disk spectra. To maintain the population of small grains and the disk's optical depth over 1-10 Myr, models may need to account for collisional fragmentation of aggregates, or condensation of dust from silicate vapor in hot, active disk regions (Dullemond \& Dominik 2005, Ormel et al. 2007). Turbulent concentration of dust (\$3.2.3) could also be an important correction to coagulation models, not only because densities are enhanced, but because collision speeds are likely reduced in particle clumps, as measured in simulations of the streaming instability (Johansen et al. 2009b, §7.2).

\section{CRITERIA FOR GRAVITATIONAL INSTABILITY}

\subsection{Dynamical Collapse}

We analyze the stability of a planar, self-gravitating, rotating sheet of dust, modeled as a fluid (GW, Safronov 1969). For the moment we ignore interactions with gas. Unperturbed, the dust has surface density $\Sigma$, angular speed $\Omega$, and barotropic pressure $P(\Sigma) \sim \Sigma c^{2}$, where $c$ is the velocity dispersion. For axisymmetric perturbations, the linearized equations for continuity and momentum read (e.g., chapter 6 of Binney \& Tremaine 2008)

$$
\frac{1}{\Sigma} \frac{\partial \Sigma^{\prime}}{\partial t}+\frac{\partial v_{r}^{\prime}}{\partial r}=0
$$




$$
\begin{array}{r}
\frac{\partial v_{r}^{\prime}}{\partial t}-2 \Omega v_{\phi}^{\prime}=\frac{-c^{2}}{\Sigma} \frac{\partial \Sigma^{\prime}}{\partial r}-\frac{\partial \Phi^{\prime}}{\partial r} \\
\frac{\partial v_{\phi}^{\prime}}{\partial t}+\frac{\kappa^{2}}{2 \Omega} v_{r}^{\prime}=0
\end{array}
$$

where perturbations are primed, $\Phi^{\prime}$ is the perturbation gravitational potential, and $\kappa=\left(r d \Omega^{2} / d r+4 \Omega^{2}\right)^{1 / 2}$ is the epicyclic frequency of radial oscillations-in practice nearly equal to $\Omega$, its value for a Kepler potential. For WKB waves, perturbations $\propto e^{\imath\left(k_{r} r+\omega t\right)}$ where $k_{r} r \gg 1$, and $\Phi^{\prime} \approx-2 \pi G \Sigma^{\prime} /\left|k_{r}\right|$ (Binney \& Tremaine 2008). Then (18)-(20) yield the well-known dispersion relation for axisymmetric waves:

$$
\omega^{2}=c^{2} k_{r}^{2}-2 \pi G \Sigma\left|k_{r}\right|+\kappa^{2}
$$

which informs us that pressure stabilizes short wavelengths, rotation stabilizes long ones, and self-gravity de-stabilizes intermediate wavelengths when

$$
Q \equiv \frac{c \kappa}{\pi G \Sigma}<1
$$

(Toomre 1964, Goldreich \& Lynden-Bell 1965). Modes whose wavelengths exceed $\lambda_{\text {crit }}=4 \pi^{2} G \Sigma / \kappa^{2}$ (neutrally stable for $c=0$ ) are always stable. The fastest growing mode has $\lambda_{\text {fgm }}=2 c^{2} /(G \Sigma)=Q^{2} \lambda_{\text {crit }} / 2$.

A standard expression for the vertical thickness of the dust layer is $h_{\mathrm{p}} \approx c / \Omega$ (caveat emptor: (16) shows this is not valid when $\mathrm{St} \ll 1$ ). Then Toomre's criterion (22) translates into a thickness criterion

$$
h_{\mathrm{p}}<h_{\mathrm{p}}^{*} \approx \frac{\pi G \Sigma_{\mathrm{p}}}{\Omega^{2}} \approx 2 \times 10^{8} F Z_{\mathrm{rel}}\left(\frac{r}{\mathrm{AU}}\right)^{3 / 2} \mathrm{~cm}
$$

or equivalently, a requirement on the midplane density

$$
\rho>\rho^{*} \approx \frac{\Sigma}{2 h_{\mathrm{p}}^{*}} \approx \frac{M_{*}}{2 \pi r^{3}} \approx 10^{-7}\left(\frac{r}{\mathrm{AU}}\right)^{-3} \mathrm{~g} \mathrm{~cm}^{-3},
$$

which has the same scaling as the Roche criterion (\$1), but is smaller by a geometric factor of $\sim 20$ that arises because the gravity of an axisymmetric ring is greater than that of a tidally distorted ellipsoid. The conditions for dynamical collapse differ by orders of magnitude from those afforded by the gas disk:

$$
\begin{aligned}
h_{\mathrm{p}}^{*} / h_{\mathrm{g}} & \approx 5 \times 10^{-4} F Z_{\mathrm{rel}}\left(\frac{r}{\mathrm{AU}}\right)^{3 / 14} \\
\rho^{*} / \rho_{\mathrm{g}} & \approx \frac{35}{F}\left(\frac{r}{\mathrm{AU}}\right)^{-3 / 14} .
\end{aligned}
$$

Turbulence would have to be exceptionally weak to allow particles to sediment to such a thin layer: from (16), $\alpha$ would need to be $\lesssim 3 \times 10^{-7} \tau_{\mathrm{s}}\left(F Z_{\text {rel }}\right)^{2}(r / \mathrm{AU})^{3 / 14}$. Overcoming this obstacle - and showing that there are alternatives to dynamical collapse from a disk of uniform Solar abundance - are the goals of this review, with some of the main ideas summarized in Figures 2 and 4 .

Sekiya (1983) solves for the linear stability of a midplane layer composed of perfectly coupled dust and gas. The layer is confined by the pressure of overlying gas layers. As with Toomre's calculation above, axisymmetry is assumed; 
however the midplane layer's vertical thickness is free to change. Dynamical instability occurs for midplane densities about 4 times greater than $\rho^{*}$ because gas pressure helps to stabilize the midplane layer. Since the layer is incompressible, in-plane motions give rise to out-of-plane "bulges." Though not modeled, slow sedimentation of dust to the centers of these bulges could produce km-scale planetesimals.

Toomre's criterion (22) is a good rule of thumb for deciding when self-gravity matters, but it misleads because it is derived for axisymmetric waves. In reality, GI proceeds non-axisymmetrically. Non-axisymmetric waves amplify when swung from leading to trailing by the background radial shear (Goldreich \& Lvnden-Bell 1965). Waves grow even if $Q>1$, but especially strongly as $Q \rightarrow 1$. Toomre (1981) gives a delightful tutorial, explaining swing amplification as a near-resonance between radial shear, epicyclic motion, and self-gravity. Amplification is restricted to the interval, of duration $\sim 1 / \Omega$, when wave pitch angles slew from about -1 to 1 rad; upon completion, $\lambda_{r} \sim \lambda_{\phi}$ and amplitudes have grown by factors $\gtrsim 50$ for $Q \lesssim 1.2$. Modes most prone to growth have $\lambda_{\phi} \approx \lambda_{\text {crit }}$. For these reasons, GW describe the disk's initial fragments as having a characteristic length $L_{\text {frag }} \sim \xi \lambda_{\text {crit }}$ and mass $M_{\text {frag }} \sim \Sigma \xi^{2} \lambda_{\text {crit }}^{2} \equiv \xi^{2} M_{\text {crit }}$, where $\xi$ is an order unity parameter that contains our uncertainty about the spectrum of seed perturbations and how close $Q$ is to unity. At $r=1 \mathrm{AU}, M_{\text {frag }}$ equates to a compact rocky planetesimal having

size $s_{\text {frag }} \approx 8\left(F Z_{\text {rel }} / 0.33\right) \xi^{2 / 3} \mathrm{~km}$; this estimate figures prominently in the lore of "kilometer-sized planetesimals."

Numerical N-body simulations of inelastically colliding particles verify that within 1-2 orbital periods, particles aggregate on the scale $\lambda_{\text {crit }}$ (Michikoshi et al. 2007). The linear mass scale $M_{\text {crit }}$ is not as prominent in either particle or gas simulations (Gammie 2001), partly because fragments accrete rapidly - suggesting that $M_{\text {crit }}$ be used cautiously, and probably as a lower limit.

\subsection{Drag-Assisted Gravitational Instability}

The stability properties discussed above pertain to dust treated as a single frictionless fluid. But gas-dust interactions can change this picture qualitatively. The simplest modification is to introduce drag terms to the momentum equations (Ward 1976, 2000, Coradini et al. 1981, Youdin 2005):

$$
\begin{array}{r}
\frac{\partial v_{r}^{\prime}}{\partial t}-2 \Omega v_{\phi}^{\prime}=\frac{-c^{2}}{\Sigma} \frac{\partial \Sigma^{\prime}}{\partial r}-\frac{\partial \Phi^{\prime}}{\partial r}-\frac{v_{r}^{\prime}}{t_{\mathrm{s}}} \\
\frac{\partial v_{\phi}^{\prime}}{\partial t}+\frac{\kappa^{2}}{2 \Omega} v_{r}^{\prime}=-\frac{v_{\phi}^{\prime}}{t_{\mathrm{s}}} .
\end{array}
$$

The meaning of $v^{\prime}$ bears clarification. With drag, particles drift radially (3.1.1). Therefore perturbed quantities refer to a background with steady $v_{r} \neq 0$, and our WKB analysis is restricted to $t<t_{r}$. Backreaction on gas, whose properties are assumed fixed, is neglected.

When $\tau_{\mathrm{s}} \ll 1$, the drag-modified equations yield a new dispersion relation:

$$
\omega=\imath\left(c^{2} k_{r}^{2}-2 \pi G \Sigma\left|k_{r}\right|\right) t_{\mathrm{s}}
$$

which implies that modes for which $k_{r}<2 \pi G \Sigma / c^{2}$ are unstable even when $Q>1$ ! The fastest growing mode has $\lambda_{\text {fgm }}=2 c^{2} /(G \Sigma)=Q^{2} \lambda_{\text {crit }} / 2$ and growth 
rate $\left|\omega_{\text {fgm }}\right|=\Omega \tau_{\mathrm{s}} / Q^{2}$. Thus drag destabilizes long wavelength modes, on longer timescales, compared to the dissipationless case. These qualitative differencesin particular the removal of Toomre's $Q$-criterion - apply for a variety of drag regimes, and for cases that include turbulent diffusion of particles, accounted for by adding $\left(D_{\mathrm{p}} / \Sigma\right) \partial^{2} \Sigma^{\prime} / \partial t^{2}$ to the right-hand side of (18) (Youdin, in preparation; Karim Shariff \& Jeffrey Cuzzi, personal communication).

Goodman \& Pindor (2000, hereafter GP00) explain the instability simply. In the unperturbed state, dust has constant surface density and drifts radially inward at constant velocity. An axisymmetric overdense ring of width $\Delta r$ exerts a gravitational pull radially inward at its outer edge. Provided $h_{\mathrm{p}} \ll \Delta r \ll h_{\mathrm{g}}$, dust rotates faster there, while the gas velocity is unaltered. The increased drag causes dust to flow at a greater rate into the annulus. Likewise there is a flow into the annulus at the inner edge, where dust experiences less drag. Thus when combined with gas drag, self-gravity, no matter how weak, can draw particles into rings.

The stability of non-axisymmetric modes with dissipation is a largely open field. Noh et al. (1991) make some exploratory integrations of the linearized perturbation equations. In N-body simulations that include $-v^{\prime} / t_{\mathrm{S}}$ drag and a fixed gas velocity field, particles cluster more readily because gas drag damps their velocity dispersion (Tanga et al. 2004).

\section{THIN DUST LAYERS: VERTICAL SHEARING INSTABILITIES}

Particles may be prevented from settling to the midplane not only by turbulence intrinsic to the gas (e.g., sustained by the MRI), but also by turbulence triggered by the particles themselves (Weidenschilling 1980). We consider here instabilities caused by small $\tau_{\mathrm{s}} \ll 1$ solids that drive a vertical shear. In 97 we show that larger particles, in addition to inducing a vertical shear, can drive turbulence of a different character via relative streaming motions.

As dust settles, a particle-rich sublayer develops which orbits at nearly the full Keplerian velocity. The dust-poor gas above and below shears by at speeds approaching the pressure-supported value $\eta v_{\mathrm{K}}$ (eq. 8). Such a stratified shear flow is subject to a Kelvin-Helmholtz-type instability (KHI). For a non-rotating flow - which we later show turns out to be a fair analogue for actual vertically shearing flows in disks - the KHI can develop when the Richardson number

$$
\operatorname{Ri}=\frac{(g / \rho) \partial \rho / \partial z}{\left(\partial v_{\phi} / \partial z\right)^{2}}<\frac{1}{4}
$$

somewhere (Drazin \& Reid 2004), where $g$ is the vertical gravitational acceleration and $\rho=\rho_{\mathrm{p}}+\rho_{\mathrm{g}}$ (see Garaud \& Lin 2004, for the applicability of Ri to a dust-gas mixture). The square root of $\mathrm{Ri}$ is the Brunt-Vaisala frequency for buoyant vertical oscillations, divided by the shear rate $\partial v_{\phi} / \partial z$. This is a sensible criterion: when the buoyancy frequency is small, vertically displaced fluid elements fail to return to their equilibrium positions before being whisked away by the background shear.

When we neglect self-gravity and approximate $g \sim-\Omega_{\mathrm{K}}^{2} h_{\mathrm{p}}$, and further take 
$\partial(\ln \rho) / \partial z \sim-1 / h_{\mathrm{p}}$ and $\left|\partial v_{\phi} / \partial z\right| \sim \eta v_{\mathrm{K}} / h_{\mathrm{p}}$, criterion (30) implies that the KHI prevents the sublayer from having a thickness less than

$$
h_{\mathrm{p}, \mathrm{Ri}} \sim \mathrm{Ri}^{1 / 2} \eta r \sim 10^{-2}\left(\frac{r}{\mathrm{AU}}\right)^{2 / 7} h_{\mathrm{g}} .
$$

Thus the midplane density falls short of $\rho^{*}$ by a factor of $\sim 20 /\left(F Z_{\text {rel }}\right)$, roughly consistent with findings by Cuzzi et al. (1993), who use mixing length models for particle-laden turbulence scaled to laboratory experiments. If we insist that the disk be of bulk Solar composition, then achieving higher densities requires either that particles be larger $\left(\tau_{\mathrm{s}} \gtrsim 1\right)$ so as to decouple from the turbulence, or that they clump locally (\$3.2.3,, 7 ).

Nominally we can estimate, using (16), that turbulence intrinsic to gas dominates the KHI when $\alpha \gtrsim \tau_{\mathrm{s}}\left(h_{\mathrm{p}, \mathrm{Ri}} / h_{\mathrm{g}}\right)^{2} \sim 3 \times 10^{-4} \tau_{\mathrm{s}}(r / \mathrm{AU})^{4 / 7}$. Whether MRIdead zones (\$2) are sufficiently passive for the KHI to manifest remains an outstanding question. Our $\alpha$ scalings may not apply to the weak motions possibly excited by MRI-active surface layers (Gammie 1996, Fleming \& Stone 2003, Oishi et al. 2007; see also Bai \& Goodman 2009 for detailed estimates of the active layer thickness).

In one of the earliest works to treat the KHI in disks realistically by accounting for orbital differential rotation, Ishitsu \& Sekiya (2003) numerically integrate the linearized perturbation equations and find that maximum growth factors increase strongly with midplane dust-to-gas ratios - so strongly that $\rho_{\mathrm{p}} / \rho_{\mathrm{g}}$ is likely to stall at values just above unity in disks having bulk solar metallicity. Their conclusion is borne out by shearing box simulations (Chiang 2008, Barranco 2009), performed in the limit that dust is perfectly coupled to gas $\left(\tau_{\mathrm{s}} \rightarrow 0\right)$. These simulations demonstrate that despite the inclusion of orbital dynamics, Richardson numbers characterizing instability remain between 0.1 and 1 (Figure 5). In retrospect this is not surprising, since rotational and radial shearing frequencies $\sim \Omega_{\mathrm{K}}$ are only of the same order as both the Brunt-Vaisala frequency, and the vertical shearing frequency $h_{\mathrm{p}, \mathrm{Ri}} /\left(\eta v_{\mathrm{K}}\right) \sim \mathrm{Ri}^{1 / 2} \Omega_{\mathrm{K}}^{-1}$, when $\mathrm{Ri} \sim 1$. Nevertheless radial shear is critical for limiting the growth of perturbations that otherwise run away when only the Coriolis force is included (cf. Gómez \& Ostriker 2005).

As pointed out by J. Goodman (personal communication; see Chiang 2008), the KHI may not be the only instability at play. Dusty sublayers may also be baroclinically unstable: their isodensity surfaces, dominated by dust, do not align with their isobars, dictated by gas (Knobloch \& Spruit 1985). Axisymmetric baroclinic stability is assured by Ishitsu \& Sekiva (2003) and by analogy with the Solberg-Hoiland criteria for rotating stars (Kippenhahn \& Weigert 1990). Specifically, displacements at constant entropy are almost perfectly radial when vertical stratification of the dust is strong. Thus in a Keplerian disk, outward adiabatic displacements move toward regions of higher specific angular momentum, and are Rayleigh stable.

Aside from postulating large, aerodynamically decoupled particles, another way to prevent the shearing instability from forestalling GI is to substantially raise $Z_{\text {rel }}$. Sekiya (1998) discovers that for flows with constant $\operatorname{Ri}(z)=1 / 4, Z_{\text {rel }} \approx 6$ 30 can lead to $\rho(z=0) \approx \rho^{*}$ in a midplane cusp. In fact, by further increasing $Z_{\text {rel }}$ slightly, and accounting properly for vertical self-gravity, the cusp becomes singular: $\rho(0) \rightarrow \infty$. YS perform similar calculations, finding $\rho \approx \rho^{*}$ could be 
achieved for $Z_{\text {rel }}$ as low as 2 , depending on disk parameters. They show that the cusp develops when $\rho_{\mathrm{p}} \gtrsim \rho_{\mathrm{g}}$ throughout the layer, which from (31) requires $\Sigma_{\mathrm{p}} / \Sigma_{\mathrm{g}} \gtrsim \sqrt{\operatorname{Ri}} \eta v_{\mathrm{K}} / c_{\mathrm{g}}$, or $Z_{\text {rel }} \gtrsim 1(r / \mathrm{AU})^{2 / 7}$. Physically, as solids are added, the buoyancy frequency increases, while the shear saturates at $\eta v_{\mathrm{K}} / h_{\mathrm{p}}$. Numerical simulations, which as yet do not include vertical self-gravity, suggest $Z_{\text {rel }} \gtrsim 5$ to attain densities approaching $\rho^{*}$ (Chiang 2008). Mechanisms to achieve supersolar metallicities are shown in Figure 2 and described in $\$ 3.1 .2$.

Whether or not they are gravitationally unstable, thin dust layers for which $\rho_{\mathrm{p}} \gtrsim \rho_{\mathrm{g}}$ are also prone to the streaming instability ( $(7.2)$, a mechanism that can further concentrate solids relative to gas when $\tau_{\mathrm{s}} \neq 0$.

\section{DRAG INSTABILITIES}

The vertical shearing instability described in $\sqrt{6}$ arises in dense particle layers, even when dust is perfectly coupled to gas $\left(\tau_{\mathrm{s}} \rightarrow 0\right)$. When relative motion between gas and dust is allowed $\left(\tau_{\mathrm{s}} \neq 0\right)$, new secular (i.e. requiring dissipation) instabilities arise. These drag instabilities can strongly clump particles relative to gas. As with vertical shear, drag instabilities require that $\rho_{\mathrm{p}} / \rho_{\mathrm{g}}$ be large enough that particles backreact significantly on gas. Self-gravity is not required, in contradistinction to the drag-assisted GI of $\$ 5.2$.

We describe two different models of drag instabilities. The secular dust layer instability (SDLI) of GP00 considers the drag arising from turbulent stresses on the stratified dust layer. The streaming instability (SI) of Youdin \& Goodman (2005, hereafter YG05) accounts for the drag forces acting mutually between gas and particles in a laminar unstratified disk. Both analytic models - as well as numerical simulations that add extra physics to the SI (Johansen et al. 2007) - show that particles clump.

\subsection{Secular Instability of the Dust Layer}

The SDLI uses a single-fluid, height-integrated, axisymmetric model of the dustrich sublayer. Dust-poor gas at the top and bottom of the sublayer shears by at relative speed $\eta v_{\mathrm{K}}$, and is envisioned to be turbulent ( 6 ). These turbulent boundary layers exert drag on the dust layer. Turbulent surface stresses are assumed to be communicated throughout the entire layer; the detailed vertical response is, by construction, ignored. To calculate the turbulent drag, GP00 adopt prescriptions from GW and Cuzzi et al. (1993) for "plate drag," drawing an analogy with Ekman flow past a rigid plate. The SDLI generates overdense rings on orbital timescales, with radial widths comparable to the sublayer vertical thickness.

The use of the plate drag formula is not well founded, except perhaps if particles in the sublayer all had $\tau_{\mathrm{s}} \sim 1$ (Youdin \& Chiang 2004). But GP00 argue that their results are more general, transcending the specific drag prescription used to obtain them. The crucial assumption underlying the SDLI is that drag is collective, i.e. it depends on the surface density of the sublayer. GP00 construct a toy model that demonstrates how any collective drag force leads to instability. The insights apply to drag instabilities generally, including the SI. 
In the toy model, the sublayer is described by a 1D distribution of mass density $\Sigma$ and velocity $v$ :

$$
\begin{aligned}
\frac{\partial \Sigma}{\partial t}+\frac{\partial}{\partial x}(\Sigma v) & =0 \\
\frac{\partial v}{\partial t}+v \frac{\partial v}{\partial x} & =g-\nu_{\mathrm{d}}(\Sigma) v
\end{aligned}
$$

where $\nu_{\mathrm{d}}(\Sigma)>0$ accounts for drag, and $g<0$ serves as a proxy for gravity, pressure, and Coriolis forces. The equilibrium state has uniform mass density $\Sigma_{0}$ and inward drift velocity $v_{0}=g / \nu_{\mathrm{d}}\left(\Sigma_{0}\right) \equiv g / \nu_{0}$. We might imagine $\nu_{\mathrm{d}}$ increases with $\Sigma$-insofar as the plate drag torque is independent of $\Sigma$ while the angular momentum of the sublayer is proportional to $\Sigma$, so that the drift speed decreases with increasing $\Sigma$-but the details are unimportant: instability arises as long as $\nu_{\mathrm{d}}$ and $g$ depend on $\Sigma$ differently. With no loss of generality, all of the $\Sigma$ dependence is relegated to $\nu_{\mathrm{d}}$.

We take linear perturbations, denoted by primes, to have a Fourier dependence $\exp (\Gamma t-\imath k x)$. The drag coefficient is expanded

$$
\nu_{\mathrm{d}}(\Sigma)=\nu_{\mathrm{d}}\left(\Sigma_{0}\right)+d \nu_{\mathrm{d}} /\left.d \Sigma\right|_{0} \Sigma^{\prime} .
$$

A non-zero drift $v_{0}$ is necessary for perturbations to $\nu_{\mathrm{d}}$ to enter linearly. The dispersion relation reads

$$
\Gamma=\frac{\nu_{0}}{2}\left(-1+2 \imath \tilde{k} \pm \sqrt{1-4 \imath \delta_{\nu} \tilde{k}}\right)
$$

where $\tilde{k} \equiv k v_{0} / \nu_{0}$ and $\delta_{\nu} \equiv d \ln \nu_{\mathrm{d}} /\left.d \ln \Sigma\right|_{0}$. For any $\delta_{\nu} \neq 0$ the positive root gives growth, since $\Re(\sqrt{1+\imath b})>1$ for all real $b$. Thus any collective drag produces instability.

To understand the growth mechanism, we examine the eigenfunctions $\Sigma^{\prime}$ and $v^{\prime}$, first Taylor expanding the growing mode about $\delta_{\nu}$,

$$
\Gamma=\Gamma_{0}+\Gamma_{1}+\Gamma_{2}=\imath k v_{0}-\imath \delta_{\nu} k v_{0}+\nu_{0} \tilde{k}^{2} \delta_{\nu}^{2},
$$

and similarly expanding $v^{\prime}=v_{0}^{\prime}+v_{1}^{\prime}+v_{2}^{\prime}$. By substituting these into the linearized eqs. (32)-(33) and equating terms of the same order, we see that $v_{0}^{\prime}=0, v_{1}^{\prime}=$ $-\delta_{\nu}\left(\Sigma^{\prime} / \Sigma_{0}\right) v_{0}$, and $v_{2}^{\prime}=-\left(\Gamma_{1} / \nu_{\mathrm{d}}\right) v_{1}^{\prime}=-\imath \tilde{k} \delta_{\nu}^{2}\left(\Sigma^{\prime} / \Sigma_{0}\right) v_{0}$. For $\delta_{\nu}=0$, oscillations are stable with wave speed $\Im\left(\Gamma_{0}\right) / k=v_{0}$. This is a neutral mode where $\Sigma^{\prime}$ simply advects with the background flow. To first order in $\delta_{\nu}, v_{1}^{\prime}$ is anti-phased with $\Sigma^{\prime}$; if $\delta_{\nu}>0$, the drift speed decreases (increases) at density maxima (minima) due to the perturbed drag force; the wave does not amplify but its speed shifts by $\Im\left(\Gamma_{1}\right) / k=-\delta_{\nu} v_{0}$. To second order in $\delta_{\nu}$, the acceleration of $v_{1}^{\prime}$ is subject to drag, which induces a secondary flow $v_{2}^{\prime}$. Since $v_{2}^{\prime}$ is $-\pi / 2$ out of phase with $\Sigma^{\prime}$, the mode grows, at rate $\Gamma_{2}$. The phase shift enters because accelerations change sign at density extrema. Figure 6 depicts the eigenfunctions.

The toy model shows that essential components of drag instabilities include the background drift $v_{0}$, which allows collective effects to enter linearly, and timedependent oscillations that drag forces overstabilize. 


\subsection{Streaming Instability}

Since the streaming instability involves a minimal amount of physics - Keplerian orbital motion, gas pressure, and a drag acceleration that is linear in relative velocity - it supports the robustness of drag instabilities. YG05 uncover the SI by modeling gas and dust as two interacting fluids that obey

$$
\begin{aligned}
\frac{D_{\mathrm{p}} \boldsymbol{v}_{\mathrm{p}}}{D t} & =-\Omega_{\mathrm{K}}^{2} \boldsymbol{r}-\frac{\boldsymbol{v}_{\mathrm{p}}-\boldsymbol{v}_{\mathrm{g}}}{t_{\mathrm{s}}} \\
\frac{D_{\mathrm{g}} \boldsymbol{v}_{\mathrm{g}}}{D t} & =-\Omega_{\mathrm{K}}^{2} \boldsymbol{r}+\frac{\rho_{\mathrm{p}}}{\rho_{\mathrm{g}}} \frac{\boldsymbol{v}_{\mathrm{p}}-\boldsymbol{v}_{\mathrm{g}}}{t_{\mathrm{s}}}-\frac{\nabla P}{\rho_{\mathrm{g}}} \\
\frac{D_{\mathrm{p}} \rho_{\mathrm{p}}}{D t} & =-\rho_{\mathrm{p}} \nabla \cdot \boldsymbol{v}_{\mathrm{p}} \\
\nabla \cdot \boldsymbol{v}_{\mathrm{g}} & =0
\end{aligned}
$$

where $D_{i} / D t \equiv \partial / \partial t+\boldsymbol{v}_{i} \cdot \nabla$. Vertical gravity is neglected, so $r$ is the cylindrical radius. Youdin \& Johansen (2007, hereafter YJ07) confirm that gas compressibility is justifiably neglected in (40), and that the fluid approximation for dust holds 3

The steady-state solutions to (37) - (40) give Keplerian motion, plus radial and azimuthal drifts between dust and gas (Nakagawa et al. 1986, \$3.1.1). The relative streaming motion is linearly overstable (YG05), behaving similarly to the GP00 toy model. The main complication is that while unstable modes can be axisymmetric, they necessarily involve motions in all three directions.

Particle clumping occurs through the backreaction term in (38): $-\rho_{\mathrm{p}}\left(\boldsymbol{v}_{\mathrm{p}}-\right.$ $\left.\boldsymbol{v}_{\mathrm{g}}\right) /\left(\rho_{\mathrm{g}} t_{\mathrm{s}}\right)$. Since $\rho_{\mathrm{g}}$ and $t_{\mathrm{s}}$ are constant-by assumption in YG05, and to excellent approximation for subsonic flows - only perturbations to $\rho_{\mathrm{p}}$ couple linearly to background streaming motions $\boldsymbol{v}_{\mathrm{p}}-\boldsymbol{v}_{\mathrm{g}}$. Thus particle clumping is required to extract energy from streaming motions, as in the GP00 toy model. The SI is ultimately powered by the background gas pressure gradient, which does work on radially flowing gas: $-v_{\mathrm{g} r} \partial P / \partial r>0$. Since streaming motions transport angular momentum inward, no energy is extracted from the Keplerian shear flow (YJ07).

The SI is controlled by two parameters: $\tau_{\mathrm{s}}$ and $\left\langle\rho_{\mathrm{p}} / \rho_{\mathrm{g}}\right\rangle_{0}$, the background dustto-gas ratio. While the SI always exists, growth is slow in the test-particle limit $\left\langle\rho_{\mathrm{p}} / \rho_{\mathrm{g}}\right\rangle \ll 1$, and in the perfect coupling limit $\tau_{\mathrm{s}} \ll 1$. As $\tau_{\mathrm{s}} \rightarrow 1$, background drift speeds peak, and modes can grow on orbital times.

The nonlinear consequences of the SI are best studied by computer simulations, which can selectively include other physics. Treating particles as a continuous fluid simplifies analytic calculations, but causes difficulties in numerical simulations. The use of artificial viscosity to avoid density discontinuities in the pressureless fluid can underestimate particle concentrations and otherwise compromise results. Alternatively, "hybrid" simulations model gas as a fluid on an Eulerian grid and solids with Lagrangian superparticles, each representing a swarm of actual particles. YJ07 simulate the linear growth of SI, and confirm

\footnotetext{
${ }^{3}$ We are not aware of a formal criterion for deciding when dust can be modeled as a fluid. The usual criteria for gas molecules, that they be collisional, do not apply to dust particles entrained in gas. YG05 suggest that coupling to the gas may suffice: if $\omega t_{\mathrm{s}} \ll 1$, then the fluid approximation is expected to be valid on timescales $\omega^{-1}$. A more rigorous criterion would be useful since many nonlinear simulations (e.g. Hogan \& Cuzzi 2007) use the two-fluid approach.
} 
that the hybrid and two-fluid approaches give convergent results. They also provide the eigenfunctions of growing Fourier modes, useful for testing codes with two-way drag forces (Balsara et al. 2009, Ishitsu et al. 2009).

Johansen \& Youdin (2007) perform nonlinear 3D simulations of the idealized SI using superparticles. Marginally coupled, $\tau_{\mathrm{s}}=1$ solids concentrate by factors of several hundred for a range of $0.2<\left\langle\rho_{\mathrm{p}} / \rho_{\mathrm{g}}\right\rangle<3.0$. Better coupled $\tau_{\mathrm{s}}=0.1$ particles give particle overdensities of several tens, but only for $\left\langle\rho_{\mathrm{p}} / \rho_{\mathrm{g}}\right\rangle>1$. When $\left\langle\rho_{\mathrm{p}} / \rho_{\mathrm{g}}\right\rangle=0.2$, particle clumping is of order unity. Sample simulations are displayed in Figure 8 .

Realistically assessing how effectively the SI can concentrate particles requires that vertical stratification be included (e.g. Johansen et al. 2006a, 2007, 2009b, hereafter JYM09), since high dust-to-gas ratios also trigger the KHI (\$6). JYM09 perform 3D hybrid simulations of $\tau_{\mathrm{s}}=0.1-0.4$ solids to study the combined effects of KHI and SI. They find a metallicity threshold for strong clumping: when $Z_{\text {rel }}$ increases from $2 / 3$ to $4 / 3$, the SI, unaided by self-gravity, triggers overdensities having $\rho_{\mathrm{p}}>10^{3} \rho_{\mathrm{g}}$. The $Z_{\text {rel }}$ threshold corresponds to $\left\langle\rho_{\mathrm{p}} / \rho_{\mathrm{g}}\right\rangle \gtrsim 1$ at the midplane, similar to the analytic criterion for the saturation of the KHI (YS). It remains to be seen if this threshold will shift, or be manifested differently, for smaller particles. Hybrid simulations with SI that include self-gravity are discussed in 98 .

\section{GRAVITATIONAL COLLAPSE INTO THE NONLINEAR REGIME}

The fragmentation of the disk into marginally self-gravitating clumps is only the beginning of the story of planetesimal formation. About six orders of magnitude need to be traversed from the Roche density to solid density. Even if clumps survive buffeting by turbulence and ram pressure stripping (Cuzzi et al. 2008), their further contraction is resisted by internal random motions and net angular momentum. Gas drag slows the sedimentation of particles toward clump centers, but it also enables such settling by braking the clump's rotation and damping random motions. For $\tau_{\mathrm{s}} \ll 1$, collapse proceeds on timescales longer than the free-fall time; how much longer is unclear. At large enough densities, inelastic collisions between particles further reduce random motions and accelerate gravitational collapse.

The popular notion that GI produces planetesimals having sizes on the order of a kilometer probably originates from GW, who recognize that a fragment having the full mass $M_{\text {frag }}$ (\$5) has too much angular momentum to collapse unimpeded to solid density. They propose instead that first-generation planetesimals form from overdense regions having horizontal sizes $\Sigma_{\mathrm{p}} r^{9 / 4} /\left(\rho_{\mathrm{s}}^{1 / 4} M_{*}^{3 / 4}\right) \ll \lambda_{\text {crit }}$; such clumps, if they conserve angular momentum, collapse into solid objects having sizes $S \sim 0.4 F Z_{\text {rel }} \mathrm{km}$, spinning just below break-up (see also $\S 15.2$ of Goldreich et al. 2004). But this estimate is unjustified because it assumes that scales $\ll \lambda_{\text {crit }}$ can collapse; this would require $Q \ll 1$, and indeed GW assume unrealistically that $c=0$.

While reliable analytic estimates of the sizes of first-generation planetesimals are lacking, numerical simulations have made great strides, providing a proof of 
principle that gravitational collapse can occur, even in turbulent flows. Johansen et al. (2007) synthesize practically all of the physics described in this review by executing 3D, self-gravitating, vertically stratified, shearing box simulations of superparticles in MRI-turbulent gas. Particles all have $\tau_{\mathrm{s}}=0.25-1$. Three ways of concentrating particles manifest: trapping within transient maxima in gas pressure ( 93.1 .2 ; the streaming instability $(\$ 7.2)$; and self-gravity. Within only a few orbital periods, the largest bound cluster of superparticles attains a mass $\sim 3 \times$ that of the 450-km-radius asteroid Ceres - though collapse to solid densities is not explicitly followed because of finite grid resolution. Surprisingly, magneto-rotational turbulence hastens gravitational collapse, through pressure trapping. The main uncertainties of these simulations are the assumption that sticking produces the largish seed particles, having sizes of $15-60 \mathrm{~cm}$ at $5 \mathrm{AU}$; and the specific realization of MRI turbulence, which depends on the unknown net magnetic flux and on microscopic dissipation parameters that are too small to simulate exactly (Lesur \& Longaretti 2007, Fromang et al. 2009, Davis et al. 2009).

MRI turbulence, though it appears to aid gravitational collapse, is not a necessary ingredient. Figure 8 displays how bound clumps also form in an unmagnetized simulation (JYM09).

\section{SUMMARY AND OUTLOOK}

For a collection of grains within a protoplanetary disk to become gravitationally bound, their density $\rho_{\mathrm{p}}$ must exceed the Roche density, which for typical parameters is over 1000 times the ambient gas density $\rho_{\mathrm{g}}$. Equivalently, the dust-to-gas ratio must be enhanced over the protosolar value by a factor of 60,000. Daunting as it may seem, the requirement may be met by several mechanisms, working in concert, that concentrate particles relative to gas.

It certainly helps to have a disk - or regions of the disk-with an abundance of solids relative to gas greater than that given by solar composition. The dustto-gas column density ratio $\Sigma_{\mathrm{p}} / \Sigma_{\mathrm{g}}$ may need to be supersolar by factors of $2-10$ (§6) see also Johansen et al. 2007) for planetesimals to form. The sharp rise in exoplanet detection rates with host star metallicity (Fischer \& Valenti 2005) reinforces the importance of initial dust abundance. Disks can also become enriched with time, as pile-ups due to radial drifts and photoevaporation of gas increase $\Sigma_{\mathrm{p}} / \Sigma_{\mathrm{g}}$ (Figure 2). Observations of gas giants are consistent with their origin in metal-enriched disks. Bulk metallicities range from $\sim 4 \times$ solar (Jupiter; 91 ) to $\sim 50 \times$ solar (HD 141569; Sato et al. 2005).

Two popular misconceptions deserve correction. First, self-gravity need not be comparable to stellar tides before its effects are felt. Gas drag on particles, abetted by arbitrarily weak self-gravity, collects particles into overdense axisymmetric rings (\$5). Second, gas turbulence does not necessarily obstruct GI. There is growing evidence that magneto-rotational turbulence creates long-lived pressure maxima that can trap particles (\$3.1.2, \$3.2).

The above concentration and enrichment mechanisms - combined with vertical settling (Figure 2) - can eventually lead to $\rho_{\mathrm{p}} / \rho_{\mathrm{g}} \sim 1$. This condition presents a much lower hurdle than the Roche criterion, or even Toomre's $Q$-criterion. Having as much inertia in particles as in gas paves the way for collective drag 
effects - notably the streaming instability - that can clump particles still further, all the way to Roche density. Figure 4 illustrates the size ladder that dust must climb to form planets, identifying key processes.

Below we chart possible courses for future work:

1. Chondrules - arguably the building blocks of first-generation planetesimalswere flash-heated in an extremely dust-rich environment, with $\rho_{\mathrm{p}}$ possibly exceeding $\rho^{*}$ (ฐ1). That none of the concentration mechanisms discussed in this review (see 3.2 .3 ) explains how they were heated indicates our story may be incomplete. In shock models for chondrules, the isothermal post-shock gas density exceeds the pre-shock density by the square of the shock Mach number, a factor of about 30-just enough for $\rho_{\mathrm{g}} \approx \rho^{*}$. This opens the exciting possibility of a violent origin for both chondrules and planetesimals, though leaves unidentified the source of such strong shocks.

2. To what extent does dust settle to the disk midplane? The answer depends on stirring by turbulence, whether driven by the solids or externally imposed. Common idealizations - notably that the dust density profile in otherwise laminar disks has constant Richardson number $\mathrm{Ri} \simeq 1 / 4$ - hold only approximately at best, as shown by simulations performed in the limit that dust and gas are perfectly coupled (Chiang 2008, Barranco 2009). Longterm evolution of the dust distribution requires relaxing the perfect coupling assumption, so that dust can sediment out of gas. Novel numerical approaches such as implicit time evolution and multiple time stepping will need development. The wide separation between stopping, orbital, and sedimentation timescales could reveal new phenomena. There may not even be a steady state to which dust settles. In laminar disks, the dust distribution may cycle through long periods of settling interrupted by brief bursts of nonuniform turbulence wherever Ri drops below unity.

3. Simulations that include the streaming instability (e.g., Johansen et al. 2007) find that particles moderately coupled to gas - having stopping times $\tau_{\mathrm{s}} \geq$ 0.1 - clump strongly enough to undergo gravitational collapse. Does the same conclusion apply to yet smaller solids, increasing the overlap between process 1 - growth by sticking - and process 3 in Figure 4]? Pessimistically, smaller solids might not settle to a layer with $\rho_{\mathrm{p}} / \rho_{\mathrm{g}} \gtrsim 1$ where streaming instabilities are most effective. Also clump lifetimes decrease with decreasing stopping time (Figure 7, 93.2.3). Optimistically, particle concentration is observed in current simulations to increase with finer grid resolution, with no convergence as yet. An adaptive mesh applied to a shearing box would be a powerful tool to track the densest particle clumps.

Together points 2 and 3 emphasize that elucidating the respective roles of sticking and self-gravity requires bridging our understanding of particle dynamics from the aerodynamically well-coupled to the marginally decoupled.

4. The post-Roche evolution of particle clusters is largely unexplored. A cluster could fragment into multiple ones, much as interstellar gas clouds undergo hierarchical fragmentation as the Jeans mass decreases with higher density. The number of fragments could be large if the initial overdensity is an azimuthally extended ringlet. A record of the initial mass function for planetesimals might even be preserved today, in the mass functions of as- 
teroids and Kuiper belt objects (Morbidelli et al. 2009). Extremely widely separated binaries (Petit et al. 2008) may be gravitationally collapsed clusters that fissioned.

We thank Xylar Asay-Davis, Joe Barranco, Jeff Cuzzi, Josh Eisner, Anders Johansen, Aaron Lee, and Paolo Padoan for discussions. Jürgen Blum, Anders Johansen, Alessandro Morbidelli, Gordon Ogilvie, and Jeff Oishi provided helpful and encouraging remarks on draft versions of this manuscript. This review owes its origin to the lunchtime talk series organized through the Berkeley Center for Integrative Planetary Sciences (CIPS). This work was supported in part by National Science Foundation grant AST-0507805.

\section{References}

1. Adachi I, Hayashi C, Nakazawa K. 1976. The gas drag effect on the elliptical motion of a solid body in the primordial solar nebula. Prog. Theor. Phys. $56: 1756-1771$

2. Alexander CMO, Grossman JN, Ebel DS, Ciesla FJ. 2008. The Formation Conditions of Chondrules and Chondrites. Science 320:1617-

3. Andrews SM, Williams JP. 2005. Circumstellar Dust Disks in Taurus-Auriga: The Submillimeter Perspective. Astrophysical Journal 631:1134-1160

4. Bai XN, Goodman J. 2009. Heat and Dust in Active Layers of Protostellar Disks. ArXiv e-prints

5. Balbus SA. 2009. Magnetohydrodynamics of Protostellar Disks. ArXiv e-prints

6. Balsara DS, Tilley DA, Rettig T, Brittain SD. 2009. Dust settling in magnetorotationally driven turbulent discs - I. Numerical methods and evidence for a vigorous streaming instability. MNRAS:804-+

7. Barranco JA. 2009. Three-Dimensional Simulations of Kelvin-Helmholtz Instability in Settled Dust Layers in Protoplanetary Disks. Astrophysical Journal 691:907-921

8. Bec J, Biferale L, Cencini M, Lanotte A, Musacchio S, Toschi F. 2007. Heavy Particle Concentration in Turbulence at Dissipative and Inertial Scales. Physical Review Letters 98:084502-+

9. Binney J, Tremaine S. 2008. Galactic Dynamics: Second Edition. Princeton University Press

10. Blum J, Wurm G. 2008. The Growth Mechanisms of Macroscopic Bodies in Protoplanetary Disks. Annual Review of Astronomy and Astrophysics 46:2156

11. Burrows CJ, Stapelfeldt KR, Watson AM, Krist JE, Ballester GE, et al. 1996. Hubble Space Telescope Observations of the Disk and Jet of HH 30. Astrophysical Journal 473:437-+

12. Calvet N, Gullbring E. 1998. The Structure and Emission of the Accretion Shock in T Tauri Stars. Astrophysical Journal 509:802-818

13. Calvet N, Hartmann L, Strom SE. 2000. Evolution of Disk Accretion. Protostars and Planets $I V: 377-+$ 
14. Cameron AGW. 1973. Accumulation processes in the primitive solar nebula. Icarus 18:407-450

15. Carballido A, Fromang S, Papaloizou J. 2006. Mid-plane sedimentation of large solid bodies in turbulent protoplanetary discs. Monthly Notices of the Royal Astronomical Society 373:1633-1640

16. Chandrasekhar S. 1987. Ellipsoidal figures of equilibrium. Dover

17. Chapman S, Cowling TG. 1970. The mathematical theory of non-uniform gases. an account of the kinetic theory of viscosity, thermal conduction and diffusion in gases. Cambridge University Press

18. Chavanis PH. 2000. Trapping of dust by coherent vortices in the solar nebula. Astronomy and Astrophysics 356:1089-1111

19. Chiang E. 2008. Vertical Shearing Instabilities in Radially Shearing Disks: The Dustiest Layers of the Protoplanetary Nebula. Astrophysical Journal 675:15491558

20. Chiang E, Murray-Clay R. 2007. Inside-out evacuation of transitional protoplanetary discs by the magneto-rotational instability. Nature Physics 3:604-608

21. Chiang EI, Goldreich P. 1997. Spectral Energy Distributions of T Tauri Stars with Passive Circumstellar Disks. Astrophysical Journal 490:368-+

22. Chiang EI, Joung MK, Creech-Eakman MJ, Qi C, Kessler JE, et al. 2001. Spectral Energy Distributions of Passive T Tauri and Herbig Ae Disks: Grain Mineralogy, Parameter Dependences, and Comparison with Infrared Space Observatory LWS Observations. Astrophysical Journal 547:1077-1089

23. Chokshi A, Tielens AGGM, Hollenbach D. 1993. Dust coagulation. Astrophysical Journal 407:806-819

24. Ciesla FJ, Cuzzi JN. 2006. The evolution of the water distribution in a viscous protoplanetary disk. Icarus 181:178-204

25. Coradini A, Magni G, Federico C. 1981. Formation of planetesimals in an evolving protoplanetary disk. Astronomy and Astrophysics 98:173-185

26. Cuzzi JN, Dobrovolskis AR, Champney JM. 1993. Particle-gas dynamics in the midplane of a protoplanetary nebula. Icarus 106:102-+

27. Cuzzi JN, Hogan RC, Paque JM, Dobrovolskis AR. 2001. Size-selective Concentration of Chondrules and Other Small Particles in Protoplanetary Nebula Turbulence. Astrophysical Journal 546:496-508

28. Cuzzi JN, Hogan RC, Shariff K. 2008. Toward Planetesimals: Dense Chondrule Clumps in the Protoplanetary Nebula. Astrophysical Journal 687:1432-1447

29. D'Alessio P, Calvet N, Hartmann L. 2001. Accretion Disks around Young Objects. III. Grain Growth. Astrophysical Journal 553:321-334

30. Davis SW, Stone JM, Pessah ME. 2009. Sustained Magnetorotational Turbulence in Local Simulations of Stratified Disks with Zero Net Magnetic Flux. ArXiv e-prints

31. Desch SJ, Ciesla FJ, Hood LL, Nakamoto T. 2005. Heating of Chondritic Materials in Solar Nebula Shocks. In Chondrites and the Protoplanetary Disk, eds. AN Krot, ERD Scott, B Reipurth, vol. 341 of Astronomical Society of the Pacific Conference Series 
32. Dominik C, Tielens AGGM. 1997. The Physics of Dust Coagulation and the Structure of Dust Aggregates in Space. Astrophysical Journal 480:647-+

33. Drazin PG, Reid WH. 2004. Hydrodynamic Stability. Cambridge University Press

34. Dubrulle B, Morfill G, Sterzik M. 1995. The dust subdisk in the protoplanetary nebula. Icarus 114:237-246

35. Dullemond CP, Dominik C. 2005. Dust coagulation in protoplanetary disks: A rapid depletion of small grains. Astronomy and Astrophysics 434:971-986

36. Dullemond CP, Hollenbach D, Kamp I, D'Alessio P. 2007. Models of the Structure and Evolution of Protoplanetary Disks. In Protostars and Planets V, eds. B Reipurth, D Jewitt, K Keil

37. Eaton JK, Fessler JR. 1994. Preferential Concentration of Particles by Turbulence. International Journal of Multiphase Flow 20:169-209

38. Epstein PS. 1924. On the Resistance Experienced by Spheres in their Motion through Gases. Physical Review 23:710-733

39. Ercolano B, Clarke CJ, Drake JJ. 2009. X-Ray Irradiated Protoplanetary Disk Atmospheres. II. Predictions from Models in Hydrostatic Equilibrium. Astrophysical Journal 699:1639-1649

40. Fischer DA, Valenti J. 2005. The Planet-Metallicity Correlation. Astrophysical Journal 622:1102-1117

41. Fleming T, Stone JM. 2003. Local Magnetohydrodynamic Models of Layered Accretion Disks. Astrophysical Journal 585:908-920

42. Frank J, King A, Raine DJ. 2002. Accretion Power in Astrophysics: Third Edition. Cambridge University Press

43. Frisch U. 1996. Turbulence. Cambridge University Press

44. Fromang S, Nelson RP. 2005. On the accumulation of solid bodies in global turbulent protoplanetary disc models. MNRAS 364:L81-L85

45. Fromang S, Papaloizou J, Lesur G, Heinemann T. 2009. Numerical Simulations of MHD Turbulence in Accretion Disks. In Astronomical Society of the Pacific Conference Series, eds. NV Pogorelov, E Audit, P Colella, GP Zank, vol. 406 of Astronomical Society of the Pacific Conference Series

46. Gammie CF. 1996. Layered Accretion in T Tauri Disks. Astrophysical Journal 457:355-+

47. Gammie CF. 2001. Nonlinear Outcome of Gravitational Instability in Cooling, Gaseous Disks. Astrophysical Journal 553:174-183

48. Garaud P, Lin DNC. 2004. On the Evolution and Stability of a Protoplanetary Disk Dust Layer. Astrophysical Journal 608:1050-1075

49. Goldreich P, Lithwick Y, Sari R. 2004. Planet Formation by Coagulation: A Focus on Uranus and Neptune. Annual Review of Astronomy and Astrophysics 42:549-601

50. Goldreich P, Lynden-Bell D. 1965. II. Spiral arms as sheared gravitational instabilities. Monthly Notices of the Royal Astronomical Society 130:125-+

51. Goldreich P, Ward WR. 1973. The Formation of Planetesimals. Astrophysical Journal 183:1051-1062 
52. Gómez GC, Ostriker EC. 2005. The Effect of the Coriolis Force on KelvinHelmholtz-driven Mixing in Protoplanetary Disks. Astrophysical Journal 630:1093-1106

53. Gooding JL, Keil K. 1981. Relative abundances of chondrule primary textural types in ordinary chondrites and their bearing on conditions of chondrule formation. Meteoritics 16:17-43

54. Goodman J, Pindor B. 2000. Secular Instability and Planetesimal Formation in the Dust Layer. Icarus 148:537-549

55. Gorti U, Hollenbach D. 2009. Photoevaporation of Circumstellar Disks By FarUltraviolet, Extreme-Ultraviolet and X-Ray Radiation from the Central Star. Astrophysical Journal 690:1539-1552

56. Greenberg R, Bottke WF, Carusi A, Valsecchi GB. 1991. Planetary accretion rates - Analytical derivation. Icarus 94:98-111

57. Guillot T. 2005. The Interiors of Giant Planets: Models and Outstanding Questions. Annual Review of Earth and Planetary Sciences 33:493-530

58. Guilloteau S, Dutrey A, Pety J, Gueth F. 2008. Resolving the circumbinary dust disk surrounding HH 30. Astronomy and Astrophysics 478:L31-L34

59. Hartmann L, D'Alessio P, Calvet N, Muzerolle J. 2006. Why Do T Tauri Disks Accrete? Astrophysical Journal 648:484-490

60. Hernández J, Hartmann L, Calvet N, Jeffries RD, Gutermuth R, et al. 2008. A Spitzer View of Protoplanetary Disks in the $\gamma$ Velorum Cluster. Astrophysical Journal 686:1195-1208

61. Hewins RH. 1996. Chondrules and the protoplanetary disk: an overview. In Chondrules and the Protoplanetary Disk, eds. RH Hewins, R Jones, E Scott. Cambridge University Press

62. Hillenbrand LA. 2005. Observational Constraints on Dust Disk Lifetimes: Implications for Planet Formation. ArXiv Astrophysics e-prints

63. Hogan RC, Cuzzi JN. 2007. Cascade model for particle concentration and enstrophy in fully developed turbulence with mass-loading feedback. Physical Review E 75:056305-+

64. Ida S, Lin DNC. 2008. Toward a Deterministic Model of Planetary Formation. V. Accumulation Near the Ice Line and Super-Earths. Astrophysical Journal 685:584-595

65. Ishitsu N, Inutsuka Si, Sekiya M. 2009. Two-fluid Instability of Dust and Gas in the Dust Layer of a Protoplanetary Disk. ArXiv e-prints

66. Ishitsu N, Sekiya M. 2003. The effects of the tidal force on shear instabilities in the dust layer of the solar nebula. Icarus 165:181-194

67. Johansen A, Henning T, Klahr H. 2006a. Dust Sedimentation and Self-sustained Kelvin-Helmholtz Turbulence in Protoplanetary Disk Midplanes. Astrophysical Journal 643:1219-1232

68. Johansen A, Klahr H, Henning T. 2006b. Gravoturbulent Formation of Planetesimals. Astrophysical Journal 636:1121-1134

69. Johansen A, Oishi JS, MacLow MM, Klahr H, Henning T, Youdin A. 2007. Rapid planetesimal formation in turbulent circumstellar disks. Nature 448:1022-1025 
70. Johansen A, Youdin A. 2007. Protoplanetary Disk Turbulence Driven by the Streaming Instability: Nonlinear Saturation and Particle Concentration. Astrophysical Journal 662:627-641

71. Johansen A, Youdin A, Klahr H. 2009a. Zonal Flows and Long-lived Axisymmetric Pressure Bumps in Magnetorotational Turbulence. Astrophysical Journal 697:1269-1289

72. Johansen A, Youdin A, MacLow MM. 2009b. Particle Clumping in Protoplanetary Disks Depends Strongly on Metallicity. ArXiv e-prints

73. Jones HRA, Butler RP, Wright JT, Marcy GW, Fischer DA, et al. 2008. A Catalogue of Nearby Exoplanets. In Precision Spectroscopy in Astrophysics, eds. NC Santos, L Pasquini, ACM Correia, M Romaniello

74. Kato MT, Nakamura K, Tandokoro R, Fujimoto M, Ida S. 2009. Modification of Angular Velocity by Inhomogeneous Magnetorotational Instability Growth in Protoplanetary Disks. Astrophysical Journal 691:1697-1706

75. Kippenhahn R, Weigert A. 1990. Stellar Structure and Evolution. Springer-Verlag

76. Knobloch E, Spruit HC. 1985. Baroclinic instability in the presence of a strong horizontal shear. Geophysical and Astrophysical Fluid Dynamics 32:197-216

77. Kusaka T, Nakano T, Hayashi C. 1970. Growth of Solid Particles in the Primordial Solar Nebula. Progress of Theoretical Physics 44:1580-1595

78. Lesur G, Longaretti PY. 2007. Impact of dimensionless numbers on the efficiency of magnetorotational instability induced turbulent transport. Monthly Notices of the Royal Astronomical Society 378:1471-1480

79. Lissauer JJ, Hubickyj O, D'Angelo G, Bodenheimer P. 2009. Models of Jupiter's growth incorporating thermal and hydrodynamic constraints. Icarus 199:338350

80. Lissauer JJ, Stevenson DJ. 2007. Formation of Giant Planets. In Protostars and Planets $V$, eds. B Reipurth, D Jewitt, K Keil

81. Lithwick Y. 2009. Formation, Survival, and Destruction of Vortices in Accretion Disks. Astrophysical Journal 693:85-96

82. Lodders K. 2003. Solar System Abundances and Condensation Temperatures of the Elements. Astrophysical Journal 591:1220-1247

83. Malhotra R. 1993. The origin of Pluto's peculiar orbit. Nature 365:819-821

84. Maxey MR. 1987. The gravitational settling of aerosol particles in homogeneous turbulence and random flow fields. Journal of Fluid Mechanics 174:441-465

85. McCabe C, Duchêne G, Ghez AM. 2003. The First Detection of Spatially Resolved Mid-Infrared Scattered Light from a Protoplanetary Disk. Astrophysical Journal Letters 588:L113-L116

86. Michikoshi S, Inutsuka Si, Kokubo E, Furuya I. 2007. N-Body Simulation of Planetesimal Formation through Gravitational Instability of a Dust Layer. Astrophysical Journal 657:521-532

87. Morbidelli A, Bottke W, Nesvorny D, Levison HF. 2009. Asteroids Were Born Big. ArXiv e-prints

88. Nakagawa Y, Sekiya M, Hayashi C. 1986. Settling and growth of dust particles in a laminar phase of a low-mass solar nebula. Icarus 67:375-390 
89. Natta A, Testi L, Calvet N, Henning T, Waters R, Wilner D. 2007. Dust in Protoplanetary Disks: Properties and Evolution. In Protostars and Planets V, eds. B Reipurth, D Jewitt, K Keil

90. Noh H, Vishniac ET, Cochran WD. 1991. Gravitational instabilities in a protoplanetary disk. Astrophysical Journal 383:372-379

91. Oishi JS, Mac Low MM, Menou K. 2007. Turbulent Torques on Protoplanets in a Dead Zone. Astrophysical Journal 670:805-819

92. Ormel CW, Cuzzi JN. 2007. Closed-form expressions for particle relative velocities induced by turbulence. Astronomy and Astrophysics 466:413-420

93. Ormel CW, Spaans M, Tielens AGGM. 2007. Dust coagulation in protoplanetary disks: porosity matters. Astronomy and Astrophysics 461:215-232

94. Pan M, Sari R. 2005. Shaping the Kuiper belt size distribution by shattering large but strengthless bodies. Icarus 173:342-348

95. Petit JM, Kavelaars JJ, Gladman BJ, Margot JL, Nicholson PD, et al. 2008. The Extreme Kuiper Belt Binary $2001 \mathrm{QW}_{322}$. Science 322:432-

96. Rice WKM, Lodato G, Pringle JE, Armitage PJ, Bonnell IA. 2004. Accelerated planetesimal growth in self-gravitating protoplanetary discs. Monthly Notices of the Royal Astronomical Society 355:543-552

97. Safronov VS. 1969. Evoliutsiia doplanetnogo oblaka. Nauka Press

98. Sato B, Fischer DA, Henry GW, Laughlin G, Butler RP, et al. 2005. The N2K Consortium. II. A Transiting Hot Saturn around HD 149026 with a Large Dense Core. Astrophysical Journal 633:465-473

99. Sekiya M. 1983. Gravitational instabilities in a dust-gas layer and formation of planetesimals in the solar nebula. Progress of Theoretical Physics 69:1116-1130

100. Sekiya M. 1998. Quasi-Equilibrium Density Distributions of Small Dust Aggregations in the Solar Nebula. Icarus 133:298-309

101. Sreenivasan KR, Stolovitzky G. 1995. Turbulent cascades. Journal of Statistical Physics 78:311-333

102. Stepinski TF, Valageas P. 1996. Global evolution of solid matter in turbulent protoplanetary disks. I. Aerodynamics of solid particles. Astronomy and Astrophysics 309:301-312

103. Stevenson DJ, Lunine JI. 1988. Rapid formation of Jupiter by diffuse redistribution of water vapor in the solar nebula. Icarus 75:146-155

104. Takeuchi T, Lin DNC. 2002. Radial Flow of Dust Particles in Accretion Disks. Astrophysical Journal 581:1344-1355

105. Tanga P, Weidenschilling SJ, Michel P, Richardson DC. 2004. Gravitational instability and clustering in a disk of planetesimals. Astronomy and Astrophysics 427:1105-1115

106. Testi L, Natta A, Shepherd DS, Wilner DJ. 2003. Large grains in the disk of CQ Tau. Astronomy and Astrophysics 403:323-328

107. Throop HB, Bally J. 2005. Can Photoevaporation Trigger Planetesimal Formation? Astrophysical Journal Letters 623:L149-L152

108. Toomre A. 1964. On the gravitational stability of a disk of stars. Astrophysical Journal 139:1217-1238 
109. Toomre A. 1981. What amplifies the spirals. In Structure and Evolution of Normal Galaxies, eds. SM Fall, D Lynden-Bell

110. Toschi F, Bodenschatz E. 2009. Lagrangian Properties of Particles in Turbulence. Annual Review of Fluid Mechanics 41:375-404

111. Voelk HJ, Jones FC, Morfill GE, Roeser S. 1980. Collisions between grains in a turbulent gas. Astronomy and Astrophysics 85:316-325

112. Vorobyov EI, Basu S. 2008. Mass Accretion Rates in Self-Regulated Disks of T Tauri Stars. Astrophysical Journal Letters 676:L139-L142

113. Ward WR. 1976. The formation of the solar system. In Frontiers of Astrophysics, ed. EH Avrett. Harvard University Press

114. Ward WR. 2000. On Planetesimal Formation: The Role of Collective Particle Behavior. In Origin of the earth and moon, edited by R.M. Canup and K. Righter and 69 collaborating authors. Tucson: University of Arizona Press., p.75-84, eds. RM Canup, K Righter, et al. University of Arizona Press

115. Watson AM, Stapelfeldt KR, Wood K, Ménard F. 2007. Multiwavelength Imaging of Young Stellar Object Disks: Toward an Understanding of Disk Structure and Dust Evolution. In Protostars and Planets V, eds. B Reipurth, D Jewitt, K Keil

116. Weidenschilling SJ. 1977a. Aerodynamics of solid bodies in the solar nebula. Monthly Notices of the Royal Astronomical Society 180:57-70

117. Weidenschilling SJ. 1977b. The distribution of mass in the planetary system and solar nebula. Astrophysics and Space Science 51:153-158

118. Weidenschilling SJ. 1980. Dust to planetesimals - Settling and coagulation in the solar nebula. Icarus 44:172-189

119. Weidenschilling SJ. 1984. Evolution of grains in a turbulent solar nebula. Icarus 60:553-567

120. Whipple FL. 1972. On certain aerodynamic processes for asteroids and comets. In From Plasma to Planet, ed. A Elvius

121. Wyatt MC. 2008. Evolution of Debris Disks. Annual Review of Astronomy and Astrophysics 46:339-383

122. Youdin A. 2008. From Grains to Planetesimals: Les Houches Lecture. ArXiv e-prints

123. Youdin A, Johansen A. 2007. Protoplanetary Disk Turbulence Driven by the Streaming Instability: Linear Evolution and Numerical Methods. Astrophysical Journal 662:613-626

124. Youdin AN. 2004. Obstacles to the Collisional Growth of Planetesimals. In Star Formation in the Interstellar Medium: In Honor of David Hollenbach, eds. D Johnstone, FC Adams, DNC Lin, DA Neufeeld, EC Ostriker, vol. 323 of Astronomical Society of the Pacific Conference Series

125. Youdin AN. 2005. Planetesimal Formation without Thresholds. I: Dissipative Gravitational Instabilities and Particle Stirring by Turbulence. ArXiv Astrophysics e-prints

126. Youdin AN, Chiang EI. 2004. Particle Pileups and Planetesimal Formation. Astrophysical Journal 601:1109-1119

127. Youdin AN, Goodman J. 2005. Streaming Instabilities in Protoplanetary Disks. Astrophysical Journal 620:459-469 
128. Youdin AN, Lithwick Y. 2007. Particle stirring in turbulent gas disks: Including orbital oscillations. Icarus 192:588-604

129. Youdin AN, Shu FH. 2002. Planetesimal Formation by Gravitational Instability. Astrophysical Journal 580:494-505 


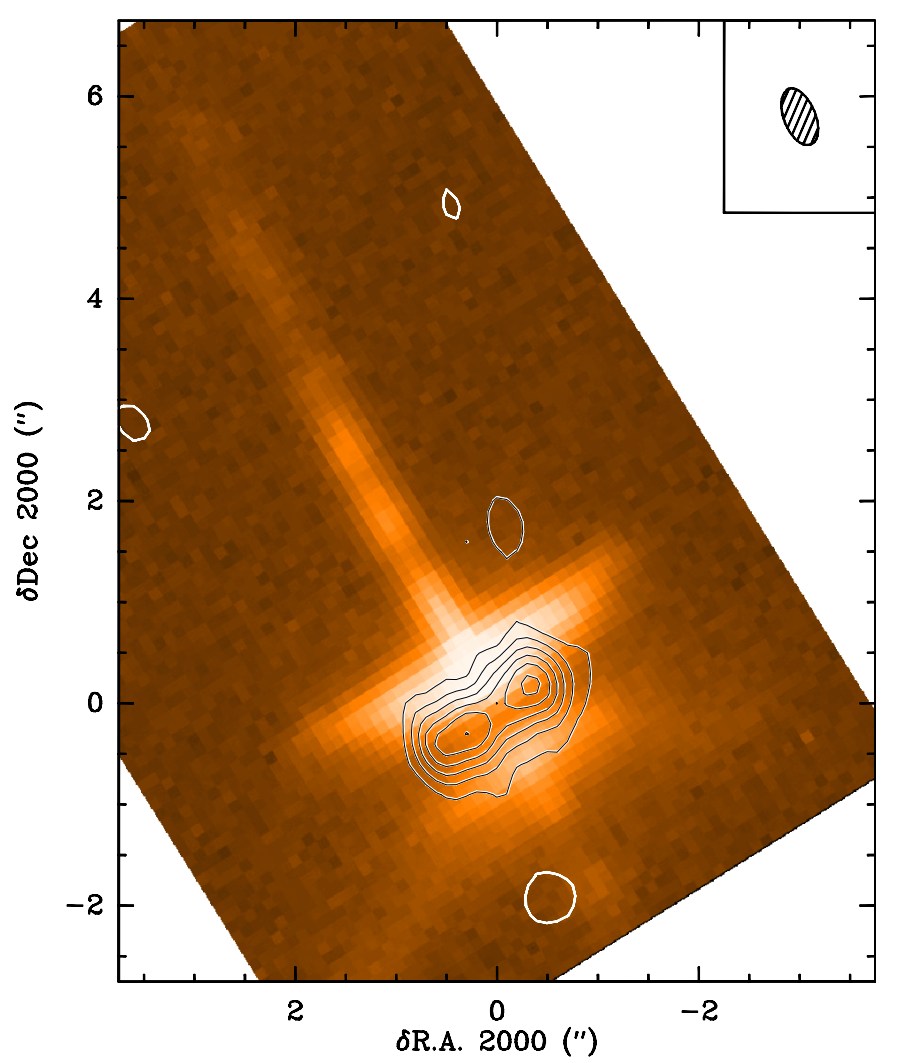

Figure 1: Hubble Space Telescope image of the protoplanetary disk HH 30, seen in scattered starlight at optical wavelengths (Burrows et al. 1996). Overlaid are contours from 1.3-mm continuum radiation measured by the Plateau de Bure Inteferometer (PdBI, Guilloteau et al. 2008). The optical image reveals the opaque disk midplane, a dark lane dividing two "bowls" of light scattered by sub- $\mu \mathrm{m}$ grains in flared disk surfaces. Perpendicular to the disk plane is the jet. Mmwave emission arises predominantly from the midplane, plausibly from $\mathrm{cm}$-sized grains that have settled vertically. The mm-wave disk has an outer radius of $\sim 130$ AU, smaller than the optical radius of $\sim 300$ AU, suggesting either that grains grow faster at smaller radius or that $\mathrm{cm}$-sized grains have drifted radially inward (3.1.1). Axes are measured in arcseconds; $1^{\prime \prime}=140 \mathrm{AU}$ for an assumed distance of 140 parsecs. The inset shaded spot shows the size of the PdBI beam, a measure of the angular resolution of the mm-wave map. Reproduced by permission of Astronomy \& Astrophysics. 

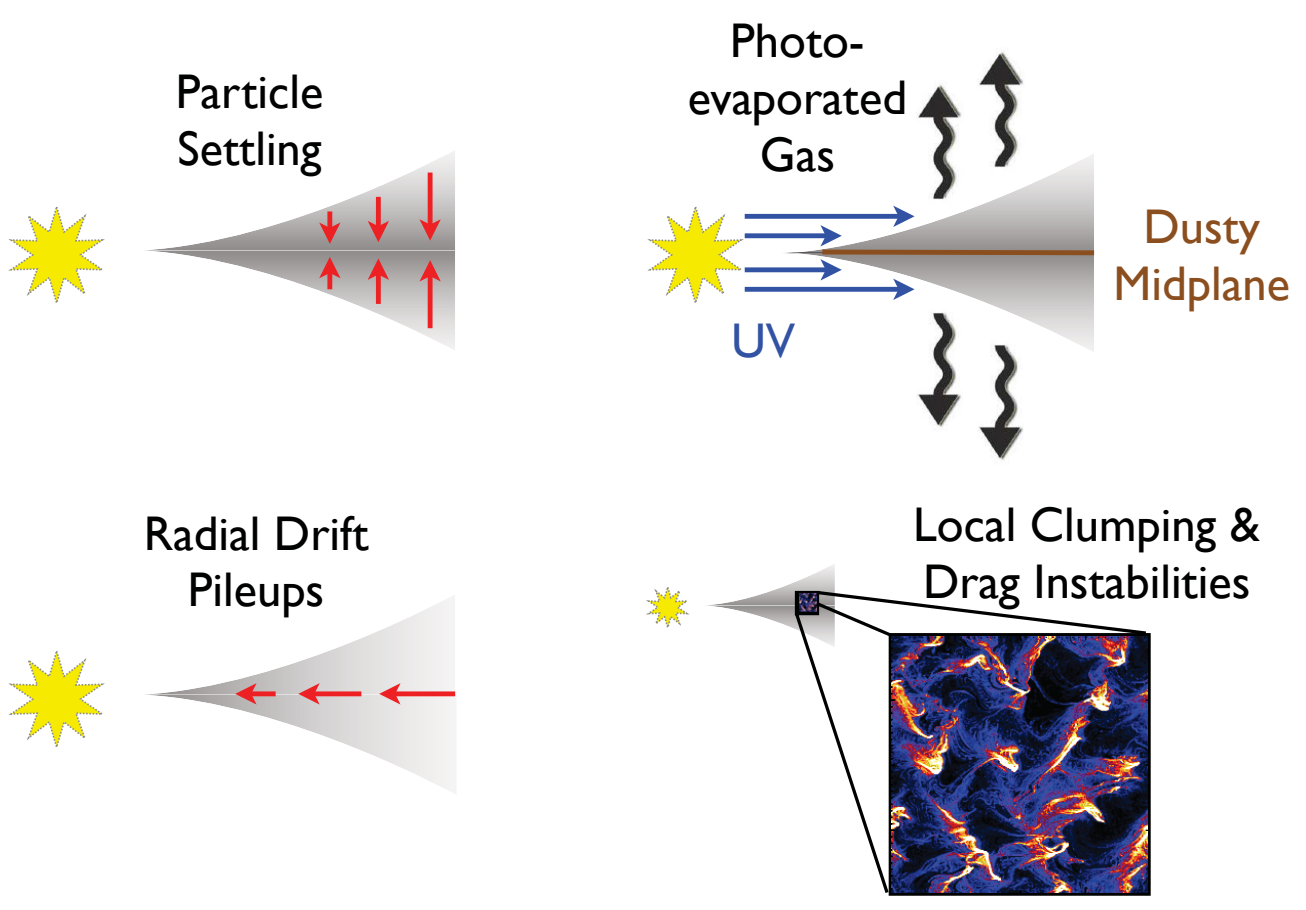

Figure 2: Four mechanisms for metal enrichment - none of which involve selfgravity. Dust can settle vertically into a dense sublayer (e.g., Chiang 2008, Barranco 2009, \$6); pile up as it drifts radially (Youdin \& Shu 2002, Youdin \& Chiang 2004, \$3.1.2); remain behind as stellar ultraviolet radiation photoevaporates gas (e.g., Throop \& Bally 2005); and be concentrated on small scales by passively responding to turbulent fluctuations (3.1.2, \$3.2.3) and by actively driving drag instabilities with gas (e.g., Goodman \& Pindor 2000, Youdin \& Goodman 2005, Johansen \& Youdin 2007, \$77). 


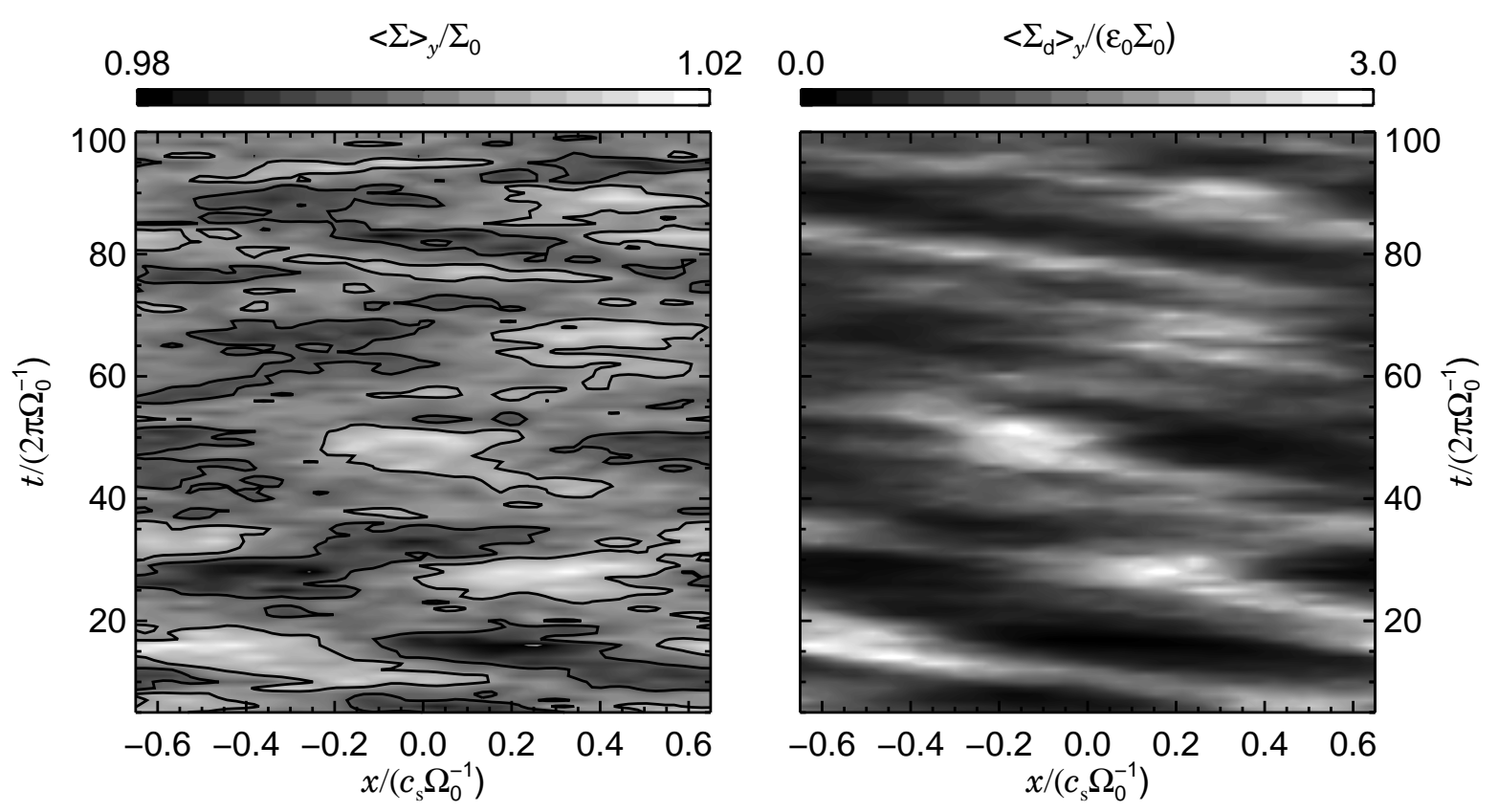

Figure 3: Test particles concentrate in turbulent pressure maxima, from 3D shearing box simulations of Johansen et al. (2006b). The surface densities of gas (left) and $\tau_{\mathrm{s}}=1.0$ solids (right) are azimuthally-averaged and plotted versus radius $x$ (in units of $h_{\mathrm{g}}$ ) and time $t$ (in orbits). Magneto-rotational turbulence drives gas fluctuations, to which particles passively respond via drag forces. The order-unity particle density fluctuations overlap the $\sim 1 \%$-scale gas density fluctuationsequivalent to the pressure fluctuations for the assumed isothermal gas. Careful inspection shows that solids collect slightly downstream (smaller $x$ ) from local pressure maxima since this is where local gradients $\partial P / \partial x>0$ cancel the global $\partial P / \partial r<0$ (see 93.1 .2$)$. Reproduced by permission of the American Astronomical Society. 


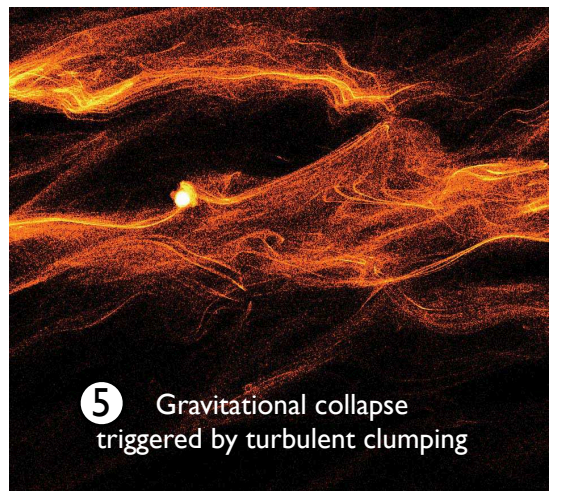

(3) Gravitational instability
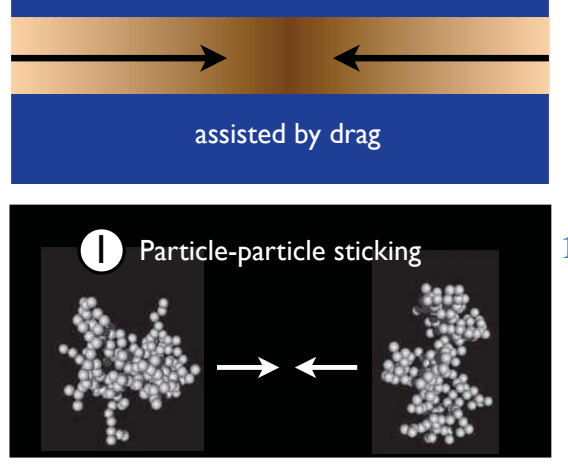

$\tau_{S}=10^{-7}$
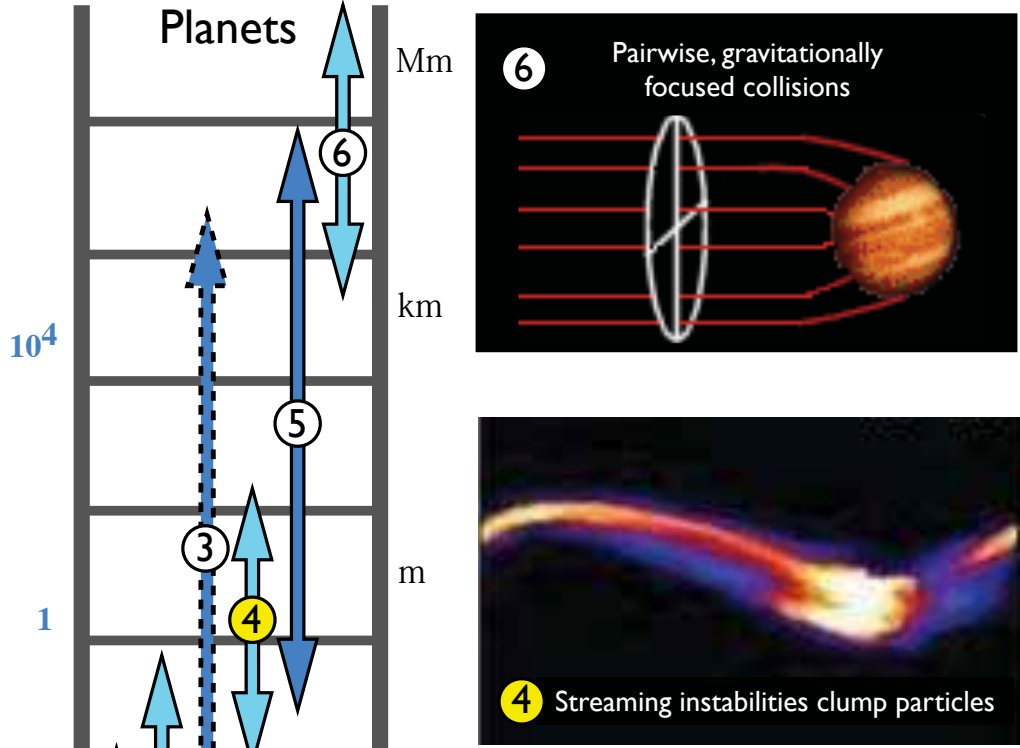

$\mathrm{mm}$

(2) Vertical settling into thin sublayers

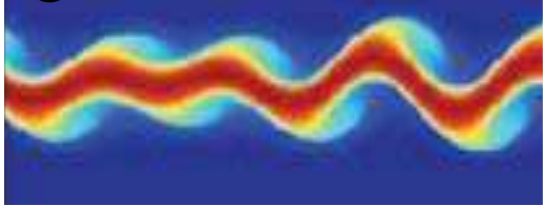

$\mathrm{S}=\mu \mathrm{m}$

Figure 4: Scaling the size ladder from dust to planets. Physical processes that either grow (labeled with white circles) or concentrate solids (yellow) are illustrated. Numbers advance from the earliest to latest stages of planet formation. The relevance of a given mechanism tends to be restricted to a certain range of particle sizes, indicated crudely by the arrows on the ladder. The ranges shown are subject to debate and actively researched. The least well explored is shown by a dashed arrow: drag-assisted GI ( $(5.2)$. Dimensionless stopping times $\tau_{\mathrm{s}} \equiv \Omega_{\mathrm{K}} t_{\mathrm{s}}$ are shown for $r=1 \mathrm{AU}$; some processes are aerodynamic and depend more on $\tau_{\mathrm{s}}$ than on particle size. 


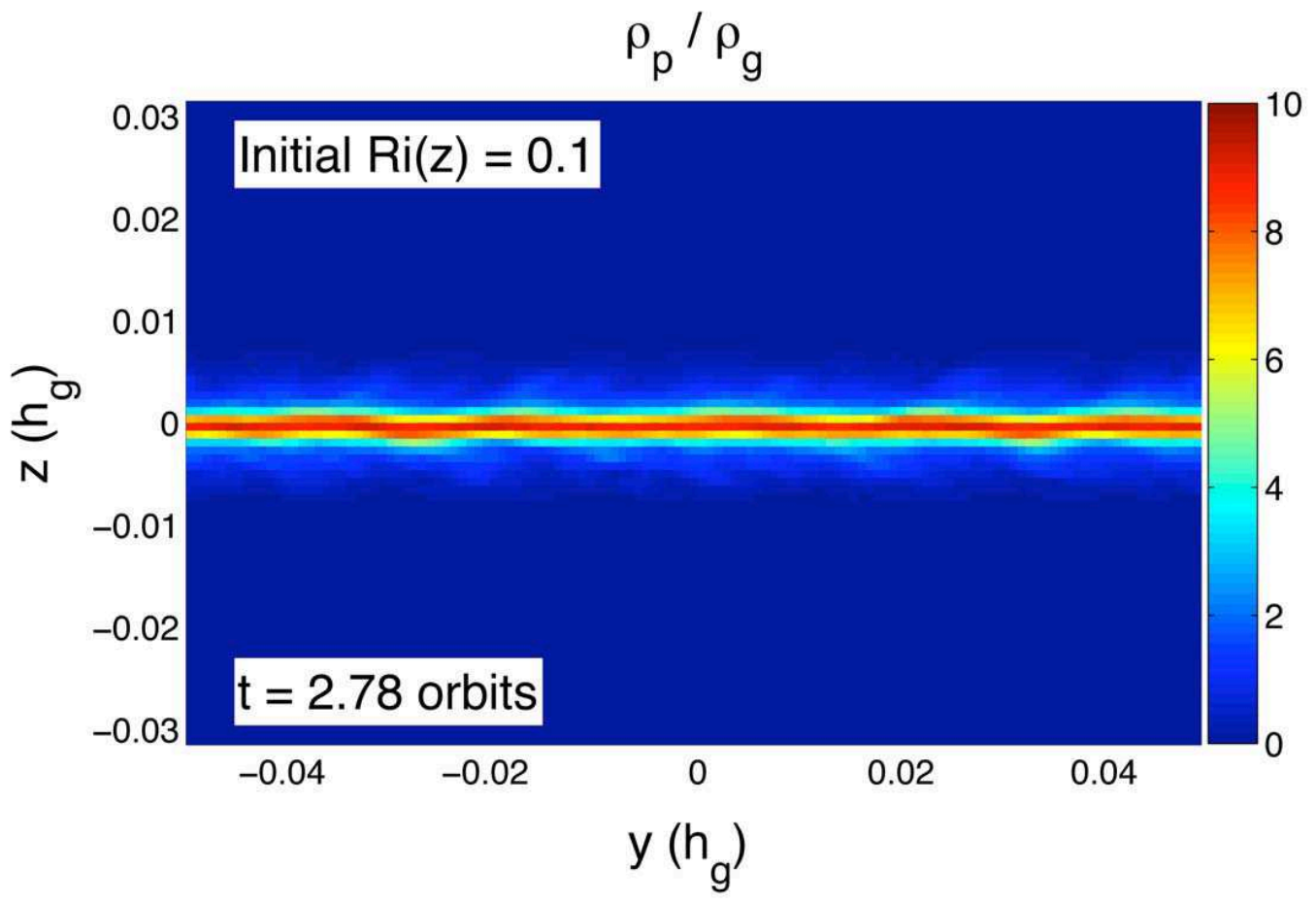

Figure 5: Dusty sublayer undergoing a Kelvin-Helmholtz-like instability, taken from a 3D shearing box simulation by Lee et al. (in prep.), using the anelastic code of Barranco (2009). The Richardson number is set initially to a constant $\mathrm{Ri}=0.1$ (Sekiva 1998), with a midplane dust-to-gas ratio of 10. Larger initial values for $\mathrm{Ri} \gtrsim 1$ produce no instability for at least 10 orbits. The vertical shearing parameter $\eta v_{\mathrm{K}} / c_{\mathrm{g}}=0.025$. The size of the shearing box is $\left(L_{x}, L_{y}, L_{z}\right)=$ $(12.8,6.35,8) z_{\max }$, where $z_{\max }$ is the maximum height of the dust layer (e.g., Chiang 2008), and the number of grid points is $\left(N_{x}, N_{y}, N_{z}\right)=(128,32,128)$. 


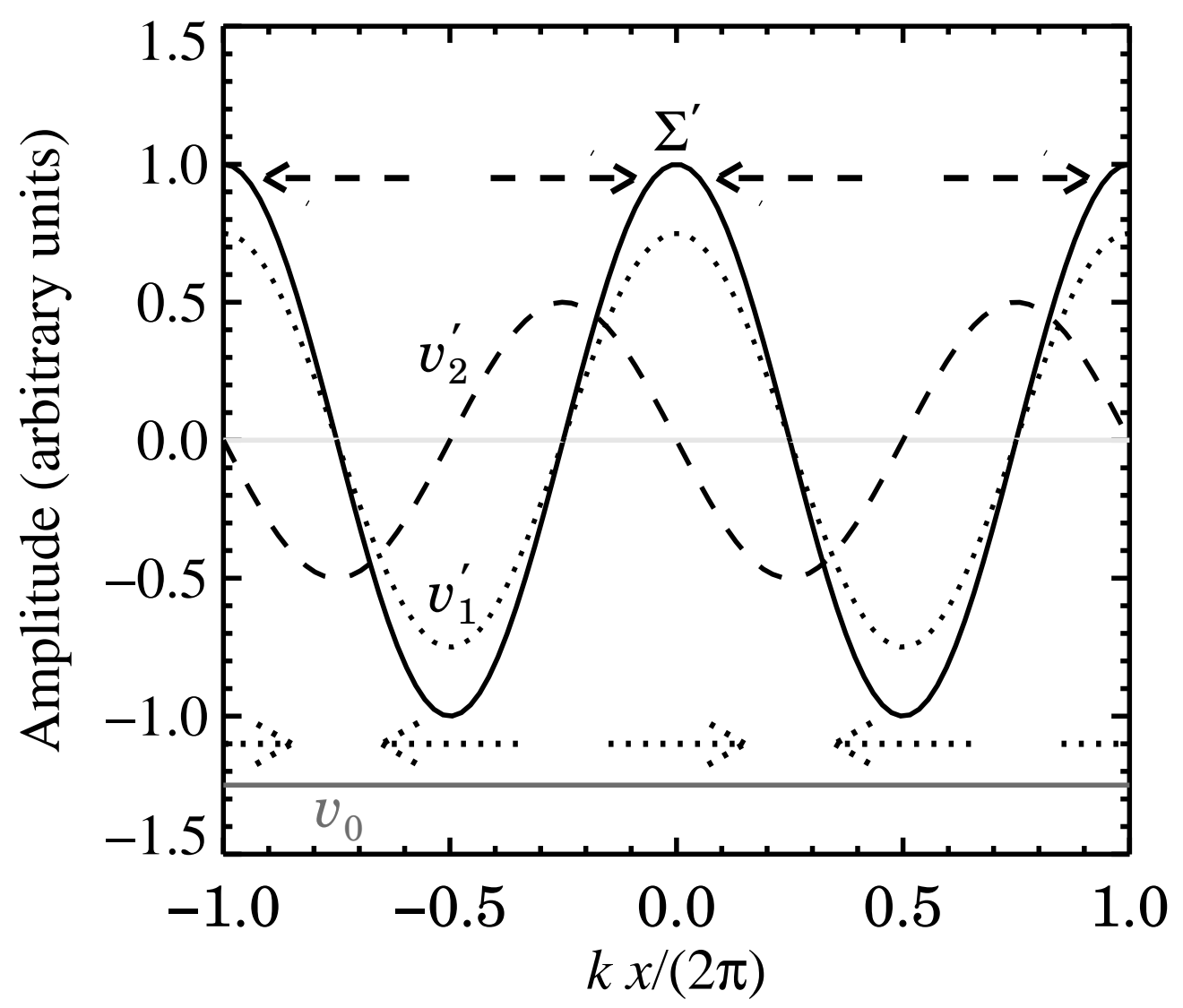

Figure 6: Mechanism behind particle clumping in drag instabilities, using the toy model of Goodman \& Pindor (2000). The equilibrium inward drift speed $v_{0}$ (grey line) is constant with local radial coordinate $x$. For linear modes, a surface density perturbation $\Sigma^{\prime}$ (solid curve) of wavelength $2 \pi / k$ has a related velocity perturbation $v^{\prime}$. To first order in $\delta_{\nu} \equiv d \ln \nu_{\mathrm{d}} / d \ln \Sigma>0$ (eqs. 33 34), overdense regions slow their drift $\left(v_{1}^{\prime}\right.$, dotted curve). Since resultant mass fluxes (dotted arrows) point toward density zeroes, there is no amplification of $\Sigma^{\prime}$ at this order; the mode merely oscillates. The second-order response $v_{2}^{\prime}$ (dashed curve) arises from drag forces acting on the first-order flow, and is phase-shifted relative to $\Sigma^{\prime}$. There are now mass fluxes (dashed arrows) that amplify the original density perturbation; the mode is overstable. 

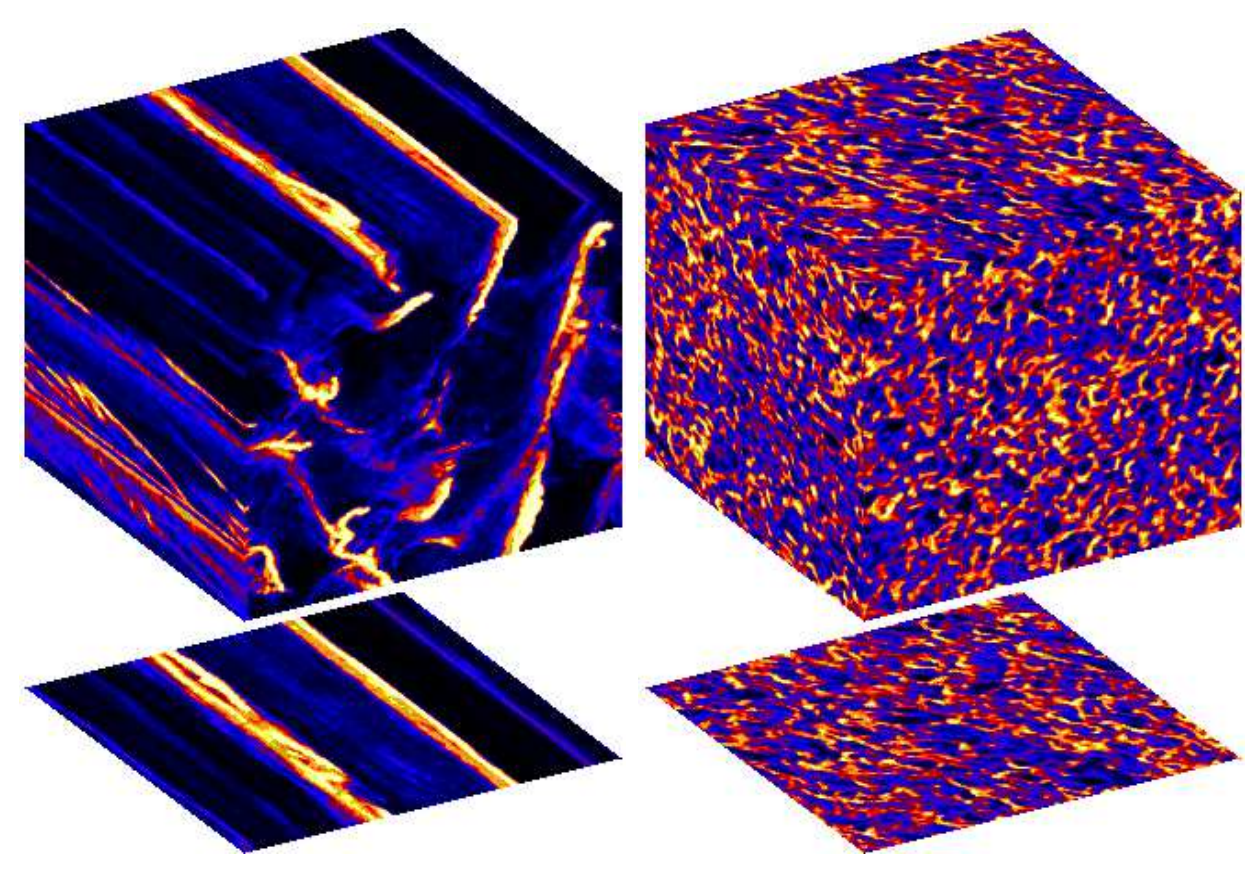

Figure 7: Saturated states of the streaming instability, from unstratified simulations of mutually coupled particles and gas by Johansen \& Youdin (2007). Shearing box axes are oriented with radius running to the right, azimuth to the left, and vertical straight up. For the simulation on the left, $\tau_{\mathrm{s}}=1$ and $\left\langle\rho_{\mathrm{p}}\right\rangle /\left\langle\rho_{\mathrm{g}}\right\rangle=0.2$; for the right, $\tau_{\mathrm{s}}=0.1$ and $\left\langle\rho_{\mathrm{p}}\right\rangle /\left\langle\rho_{\mathrm{g}}\right\rangle=1$. Colors show $\rho_{\mathrm{p}}$ at the sides of the box, with black corresponding to zero density and bright yellow corresponding to densities up to $\sim 30 \rho_{\mathrm{g}}$ (left) or $\sim 10 \rho_{\mathrm{g}}$ (right). Both snapshots are taken tens of orbits after the SI has saturated, when turbulence is fully developed. For $\tau_{\mathrm{s}}=1$, clumps are long-lived, so that Keplerian shear smears them into rings. Clumps of more tightly coupled particles are shorter-lived. Higher resolution simulations, which are feasible in $2 \mathrm{D}$, produce still stronger clumping. Reproduced by permission of the American Astronomical Society. 


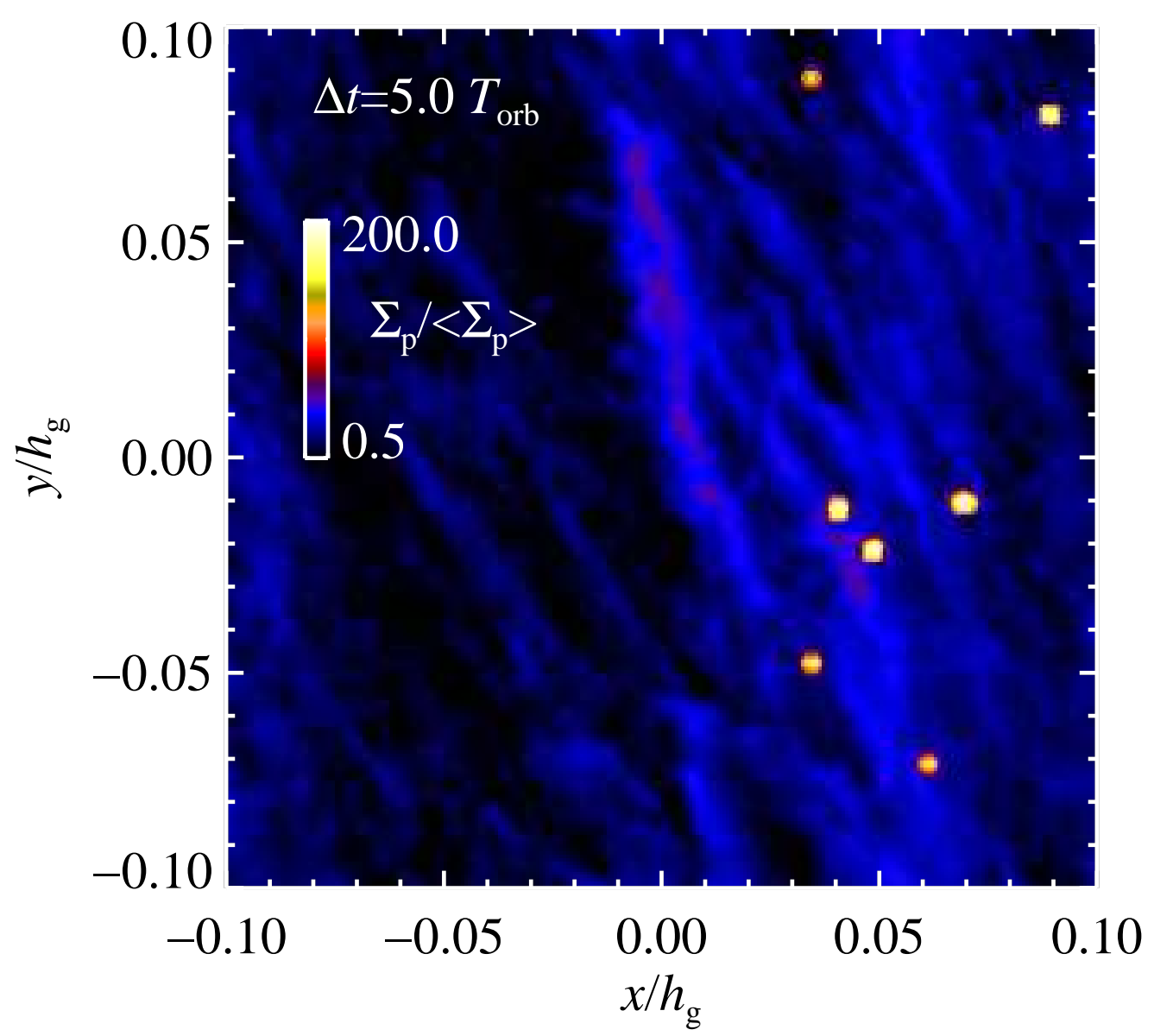

Figure 8: Particle column density $\Sigma_{\mathrm{p}}$, showing the formation of seven gravitationally bound clumps in a $3 \mathrm{D}$, vertically stratified, shearing box simulation of unmagnetized gas and superparticles with $\tau_{\mathrm{s}}=0.1-0.4$ (Johansen et al. 2009b) The box-averaged $\left\langle\Sigma_{\mathrm{p}}\right\rangle /\left\langle\Sigma_{\mathrm{g}}\right\rangle=0.02$. The $x(y)$ axis is parallel to the radial (azimuthal) direction, and measured in units of the gas scale height. The simulation first evolved for 40 orbits without self-gravity, during which particles settled to the midplane and triggered vertical shearing and streaming instabilities; strong clumping resulted. This snapshot is taken 5 orbits after self-gravity was turned on. The bound fragments contain $\sim 20 \%$ of the total mass in solids; each has a mass comparable to a compact planetesimal having a size $100-200 \mathrm{~km}$. 